\title{
Multi-Axis Identifiability Using Single-Surface Parameter Estimation Maneuvers on the X-48B Blended Wing Body
}

\author{
Nalin A. Ratnayake*, Ed T. Koshimoto, and Brian R. Taylor ${ }^{\ddagger}$
}

NASA Dryden Flight Research Center, Edwards AFB, CA 93523.

The problem of parameter estimation on hybrid-wing-body type aircraft is complicated by the fact that many design candidates for such aircraft involve a large number of aerodynamic control effectors that act in coplanar motion. This fact adds to the complexity already present in the parameter estimation problem for any aircraft with a closed-loop control system. Decorrelation of system inputs must be performed in order to ascertain individual surface derivatives with any sort of mathematical confidence. Non-standard control surface configurations, such as clamshell surfaces and drag-rudder modes, further complicate the modeling task. In this paper, asymmetric, single-surface maneuvers are used to excite multiple axes of aircraft motion simultaneously. Time history reconstructions of the moment coefficients computed by the solved regression models are then compared to each other in order to assess relative model accuracy. The reduced flight-test time required for inner surface parameter estimation using multi-axis methods was found to come at the cost of slightly reduced accuracy and statistical confidence for linear regression methods. Since the multi-axis maneuvers captured parameter estimates similar to both longitudinal and lateral-directional maneuvers combined, the number of test points required for the inner, aileron-like surfaces could in theory have been reduced by $50 \%$. While trends were similar, however, individual parameters as estimated by a multi-axis model were typically different by an average absolute difference of roughly 15-20\%, with decreased statistical significance, than those estimated by a single-axis model. The multi-axis model exhibited an increase in overall fit error of roughly 1-5\% for the linear regression estimates with respect to the single-axis model, when applied to flight data designed for each, respectively.

\section{Nomenclature}

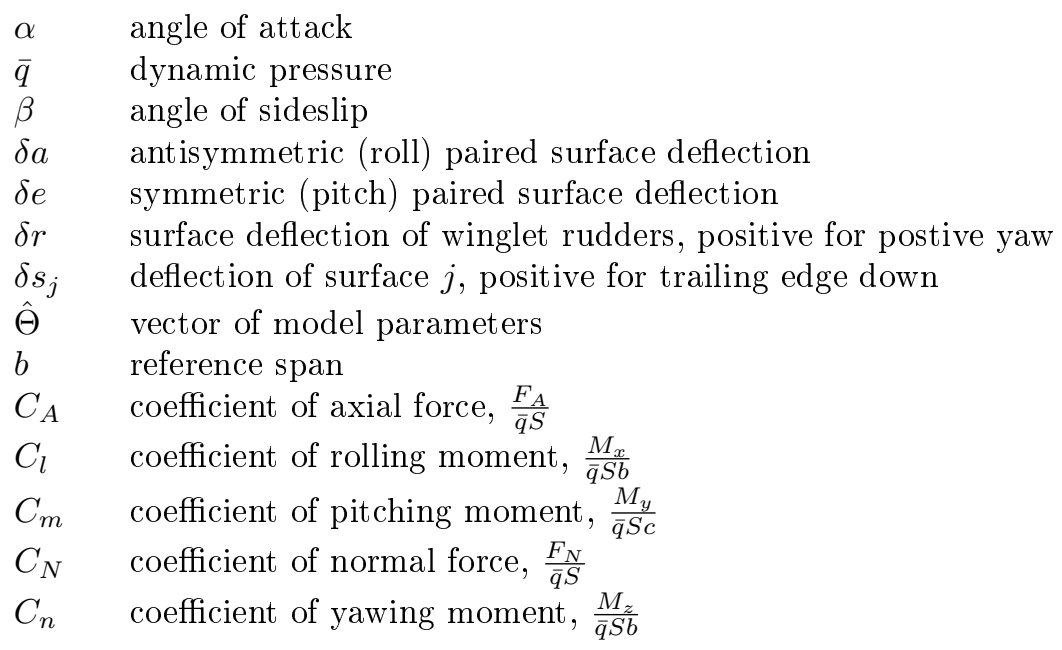

*Aerospace Engineer, Aerodynamics and Propulsion Branch, M/S 4840B, AIAA Senior Member.

${ }^{\dagger}$ Aerospace Engineer, Aerodynamics and Propulsion Branch, M/S 2228-D.

${ }^{\ddagger}$ Aerospace Engineer, Controls and Dynamics Branch, M/S 4840D, AIAA Member. 


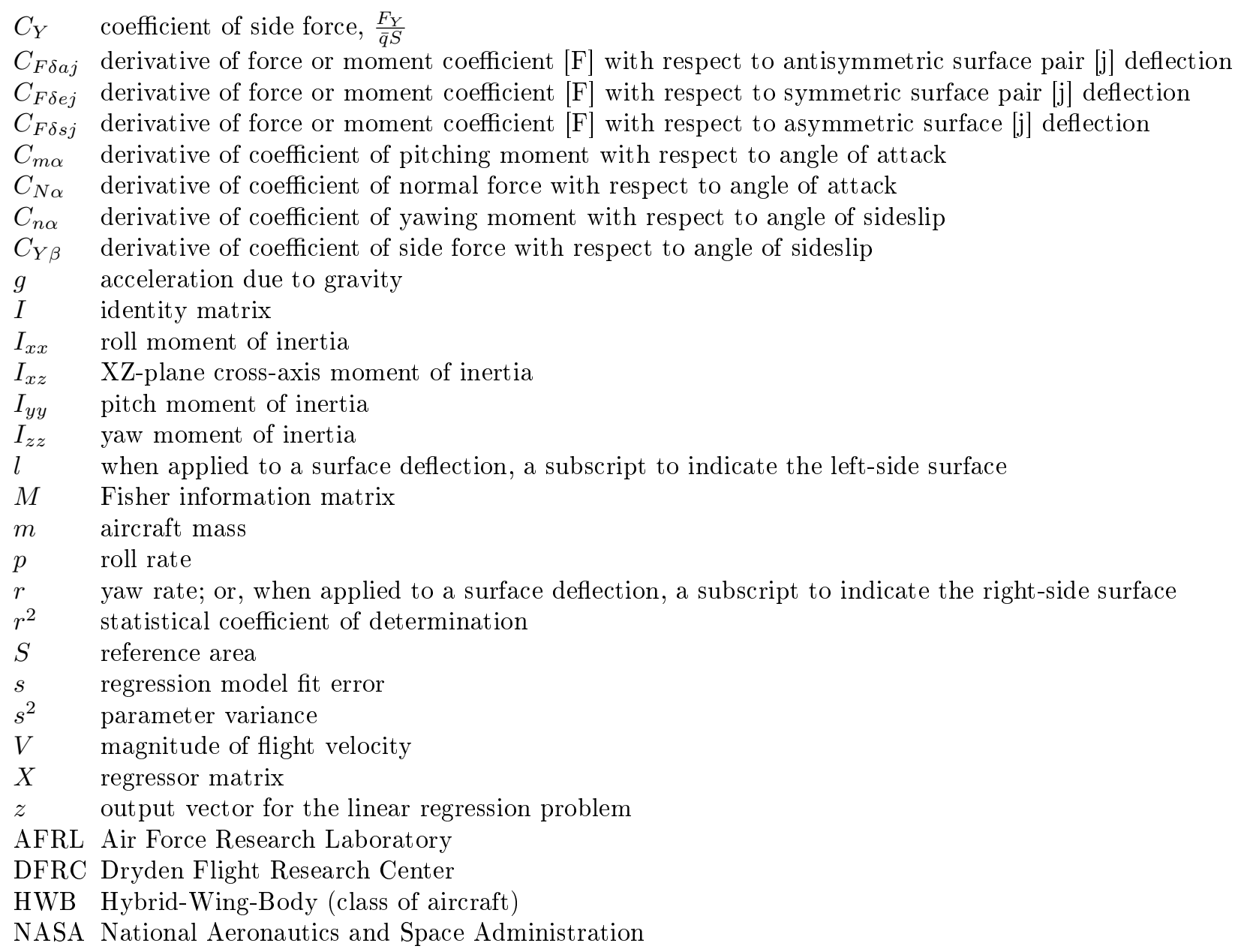

\section{Introduction}

The X-48B Blended Wing Body is an 8.5\% dynamically-scaled, hybrid-wing-body (HWB) aircraft built by Cranfield Aerospace Ltd (United Kingdom); it was the result of a joint partnership between the National Aeronautics and Space Administration (NASA), the Air Force Research Laboratory (AFRL), and The Boeing Company (Chicago, Illinois). The X-48B and its planned successors are representative of possible future, highly efficient, HWB transport designs that involve integrated configurations using a large number of control effectors. The Environmentally Responsible Aviation project within the NASA Aeronautics Research Mission Directorate intends to use the conclusions drawn from flight research on a series of configurations for the X-48B to study the development of new, manned, highly-efficient, HWB transport aircraft. A photograph of the X-48B in flight at the NASA Dryden Flight Research Center (DFRC) (Edwards, California) is shown in Fig. 1.

Research is being conducted at DFRC to study the effectiveness of various parameter estimation methods on HWB aircraft. The problem of parameter estimation on HWB aircraft is complicated by the fact that many design candidates for such aircraft involve a large number of aerodynamic control effectors that act in coplanar motion. This adds to the complexity already present in the parameter estimation problem for any aircraft with a closed-loop control system. Decorrelation of system inputs must be performed in order to ascertain individual surface derivatives with any sort of mathematical confidence. Non-standard control surface configurations, such as clamshell surfaces and drag-rudder modes, further complicate the modeling task.

A previous paper ${ }^{1}$ that studied the efficacy of single-axis, doublet-based parameter estimation maneuvers in the longitudinal axis was published by two of the present authors in early 2010 . A paper ${ }^{2}$ on the lateraldirectional results using these same methods was published in 2011. These two papers establish a set of results using single-axis methods that were used as the baseline for the present study. 


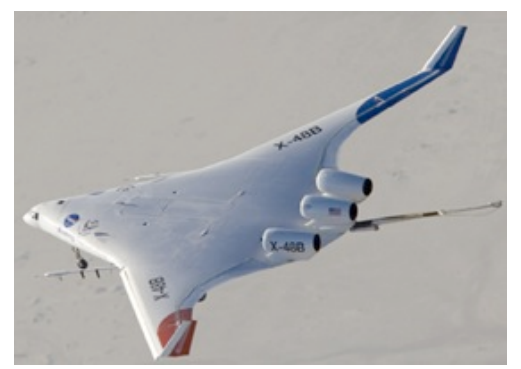

Figure 1. The Boeing X-48B Blended Wing Body, shown in flight near the NASA Dryden Flight Research Center, Edwards, California.

The present paper focuses on indentifiability considerations of performing simultaneous parameter estimation for all three axes of motion using a single-surface maneuver. Such maneuvers will be referred to as "multi-axis" maneuvers. Multi-axis maneuvers carry the benefit of decreased flight-test time required, since multiple parameters may be solved for using the same maneuver. The tradeoff between reduced flight-test time and model accuracy is assessed in this study for the inner, elevon-like surfaces of the X-48B Blended Wing Body.

The nature of the tradeoff between flight-test time and parameter estimation quality may be of interest to flight programs for which the amount of total testing is constrained below technically desirable levels by financial or operational considerations.

\section{Aircraft Description}

The X-48B aircraft incorporates a unique configuration and outer mold line. Instruments relevant to parameter estimation include dual airdata probes to measure airspeed, angle of attack, and angle of sideslip. Additionally, the aircraft is equipped with an Inertial Measurement Unit (IMU) and Global Positioning System (GPS) that provides linear acceleration, angular rotation rates, Euler angles, and position. Twenty actuating aerodynamic surfaces, eighteen of which are coplanar, are used to provide aircraft control.

The X-48B aircraft can be configured with the leading edge slats extended or retracted; however, they cannot be adjusted in flight. The center of gravity can be adjusted on the ground between forward and aft configurations. Allocation of the control surfaces is depicted in Fig. 2 with surface pairs numbered for reference. The inner surfaces (1 through 5 ) are elevons. Surfaces 6 and 7 are split ailerons, or "clamshell" surfaces: the top and bottom surface can be moved together to produce roll moments or they can be split to produce a yaw moment through differential drag. Rudders are incorporated into the winglets to provide additional yaw control and stability.

Control surface positions are inferred from the measured actuator position and are not measured directly. The control surface actuation on the X-48B aircraft consists of an electro-mechanical servo that moves the control surface through a linkage. Position measurement is taken at the output shaft of the servo; thus, differences between the surface position and actuator position may be due to linkage bending or gear slop. No corrections were made to the control surface data because data or models necessary for corrections were not available.

While these factors complicate any attempt to make absolute quantification of the individual parameters, much work can be done in studying the relative effectiveness of parameter estimation techniques and the associated model structure. Present experimental work by NASA is ongoing to attempt to quantify the uncertainty in the control surface position, as well as the propagated effect into the estimated parameters. This paper applies the models suggested by stepwise regression to simulation and flight data for the X-48B aircraft, analyzes trends in the Cramér-Rao lower bounds, assesses the fit of state-variable time history reconstructions from the solved parameters, and verifies the stepwise regression model selection by comparison to possible alternatives. 


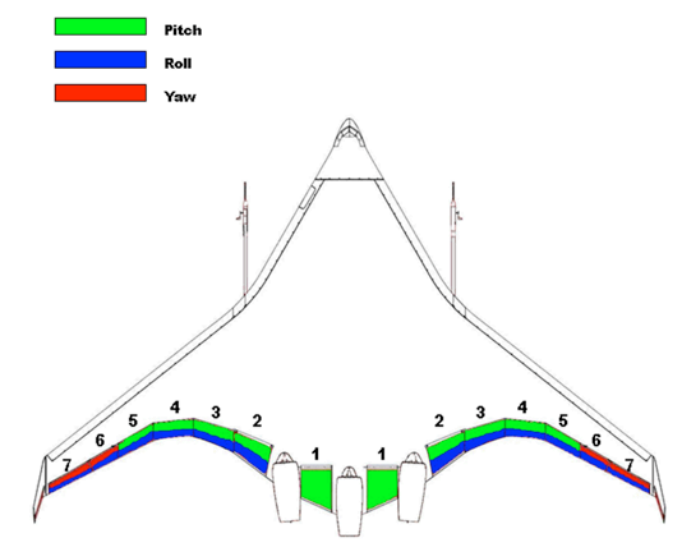

Figure 2. Control surface numbering and axis allocation for the X-48B Blended Wing Body.

\section{Method}

Parameter estimation is a subset of the broader field of system identification, wherein the basic task of the engineer is to determine the nature of a system under study through observation and analysis of the outputs generated by a controlled set of inputs. ${ }^{3}$ Parameter estimation assumes that the system in question may be modeled as a parametrized set of equations, of which the coefficients, or parameters, are the objective of the analysis.

The generally accepted standard method of performing maneuvers for parameter estimation are the traditional doublet or triplet inputs. The pilot inputs a simple square wave command of controlled magnitude in a particular axis, and the output dynamics that result from this input are analyzed for a mathematical relationship. In the case of multiple surfaces affecting the same axis, in theory, each surface and associated surface (its opposite pair on the other wing, as well as adjacent surfaces that may provide interference effects) must be tested in all possible combinations for comprehensive model validation. On aircraft with a high number of surfaces, this can be quite time-consuming.

\section{A. Linear Regression for Parameter Estimation}

Linear regression parameter estimation is a technique in which the coefficients of an assumed linear relationship between known inputs and observed outputs are estimated using least-squared fits. More information on linear regression parameter estimation techniques can be found in Klein and Morelli. ${ }^{4}$ Linear regression is often compared to output-error techniques, which actually integrate the equations of motion and compare the state outputs to measured values in order to estimate the parameters. In this sense, linear regression is mathematically simpler and therefore quicker in terms of computational time and complexity. The full derivation of such techniques is beyond the scope of this paper. More information on output-error and maximum likelihood techniques can be found in many available references on the subject. ${ }^{3,5-8}$ Similarly, there are many references ${ }^{9-15}$ that describe prior work to decorrelate control surfaces, including substantial work by Morelli and his colleagues in the field of optimal input design.

A tool often used to quantify the relative statistical confidence of a parameter estimate is the CramérRao lower bound. The Cramér-Rao lower bound represents the lowest magnitude limit for the variance of an estimator with a given bias ${ }^{16}$. Un-modeled dynamics can make the true value of the variance of the estimator much higher. In the simplest case where the variance is assumed to be unbiased and have a normal distribution, the Cramér-Rao bound becomes simply the inverse of the Fisher information matrix, $M\left(\xi_{t}\right)$, which is a metric for measuring the amount of usable information content in a set of data. Choosing input design methods and flight-test techniques that lower the Cramér-Rao bounds is an effective approach to 
choosing inputs that maximize the usable information content of the flight data.

The derivation of the Cramér-Rao bound assumes that the residuals consist solely of white noise. In practice, this is not the case. As a result, traditionally-computed bounds can be inaccurate. The CramérRao bounds presented in this report have been adjusted to account for frequency content in the residuals, using a technique from Klein and Morelli. ${ }^{4}$

\section{B. Data Sources}

Time histories of the aircraft angular rates, air data, control surface position, and other pertinent information for performing parameter estimation problems were obtained from two sources: the nonlinear simulation provided by The Boeing Company (Chicago, Illinois), and recorded data from the Phase 1 and Phase 1.5 series of flight tests of the X-48B aircraft at NASA DFRC. For both simulation and flight, force and moment coefficients were constructed from observed air data and flight dynamics using standard aircraft equations of motion.

\section{A. Maneuver Description}

For the standard, single-axis maneuvers, a series of symmetric (equal magnitude in the same direction, inducing pure pitch) or antisymmetric doublets (equal magnitude in opposite directions, inducing pure roll) was performed in both simulation and flight. For the simulation studies, the surface motions could be performed serially in a single combined maneuver termed a supermanuever, on which regression could be performed. An example is shown in Figure 3.

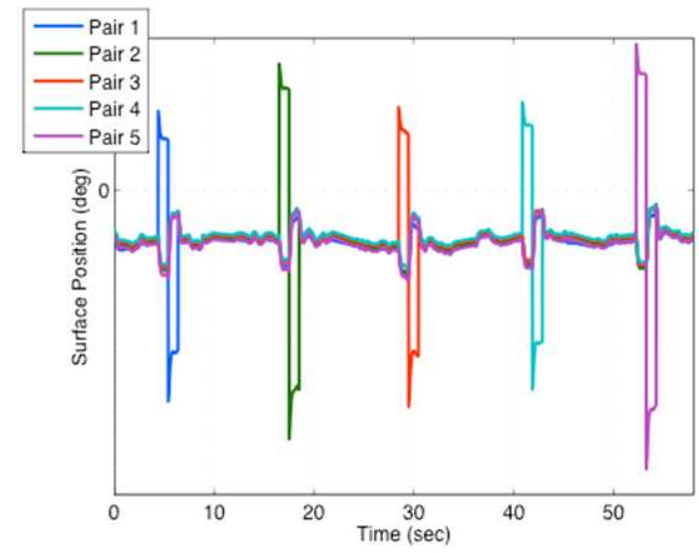

Figure 3. Multi-surface supermaneuver for temporal separation.

It is worthwhile to note that the use of supermaneuvers means that every surface excitation in a sequence is present in the dataset for all parameter estimate regression solutions, regardless of which parameters are the focus of the particular model being solved. For more details on the single-axis parameter estimation method and results for the X-48B aircraft, see Taylor and Ratnayake ${ }^{1}$ for the longitudinal results, and Ratnayake, Waggoner, and Taylor ${ }^{2}$ for the lateral-directional results.

For multi-axis / single-surface maneuvers, similar supermanuevers were constructed for the inner five surfaces, but using only a surface on one wing instead of using paired symmetric or antisymmetric maneuvers. This asymmetry induces motion in multiple axes simultaneously. Parameter estimation for all surfaces under consideration was then performed on the single combined supermaneuver.

\section{B. Flight-Testing}

Flights 65, 66, and 67 from Phase 1 of the X-48B flight-test program included standard, single-axis, doublet lateral-directional parameter estimation test points for the present analysis, at angles of attack of $6 \mathrm{deg}, 8$ deg, and $10 \mathrm{deg}$, respectively. Flights 85 and 89 from Phase 1.5 of the program included single-surface / multi-axis maneuvers at the same angles of attack. The range of altitudes and corresponding trim speeds for these angles of attack do not vary enough for a second variable to be considered in defining the flight 
condition. Individual surface pair maneuvers were available for analysis. Each maneuver was performed three times in flight in order to reduce error and quantify the variance of the dataset.

While smoothing was applied to the transition points to eliminate any discontinuities, two additional factors ameliorated the effect of splicing on the parameter estimation results. First, linear regression, as an equation-error technique, does not rely on integrating the equations of motion as in output-error techniques. This means that there is no need to ensure that integrators are properly reset and that data is exactly aligned at the splicing points for the parameter estimation (though it is required later for time-history reconstruction). Second, the range of flight conditions and possible trim states for the X-48B aircraft is limited enough that significant disparities in state variables for the same trim angle of attack are unlikely.

Noise present in the flight data was removed using a third-order, two-way Butterworth filter applied with a corner frequency of $5 \mathrm{~Hz}$, because this cutoff appeared to capture the dynamics of interest while excluding the bulk of the measurement and physical noise. Corrections were also made to measured air data as well as translational accelerations to account for the distance from the aircraft center of gravity to the respective measurement points. The airdata is thus corrected for upwash and sidewash induced by the rotation rates of the aircraft.

\section{Nonlinear Simulation}

The nonlinear simulation of the $\mathrm{X}-48 \mathrm{~B}$ aircraft was designed by The Boeing Company and is implemented in Simulink@ (The MathWorks, Natick, Massachusetts). The version of the simulation used for this paper was 4.3.1, using Vehicle Management System (VMS) version 4.3 and aerodynamic model 20100527. Note that this is a more recent aerodynamic model version than used in previous work that was performed on Phase 1 data only. ${ }^{1,2}$

Though flight is the only aeronautical reality, the advantage of conducting simulation studies is that various real-world effects can be controlled or are often simply not modeled, and the isolated effects of various external factors on the results can be explored. The simulation results shown in this paper were for supermaneuvers performed with the flight control laws forced into an open-loop mode. Further, the normal control surface allocator was bypassed in order to insert specific input combinations that were not available in the normal control laws. Applying the parameter estimation method on the simulation data also allows for consistency checks on the method.

The simulation maneuvers were initially planned for the same angles of attack as the flight data. It became necessary, however, to move the simulation points to take place at trim angles of attack on the halfangle (for example, $6.5 \mathrm{deg}, 7.5 \mathrm{deg}$, et cetera) in order to avoid breakpoints in the aerodynamic model, which were presumably artifacts from the wind-tunnel test procedure. These breakpoints made finite difference approximations in their vicinity difficult, which affects related research that would use the same data.

\section{Effector Definitions}

The configuration of the X-48B aircraft allows for several possible definitions of the control effector regressor functions due to the split nature of the clamshell surfaces. Because the clamshell surfaces can move in unison or oppositely, the same clamshell upper and lower surface can behave like a traditional aileron (primarily affecting roll), or split open in a drag-rudder yaw mode.

Deflection of any individual surface will be denoted as $\delta s$; for example, the deflection of the inner surfaces will be represented by $\delta s_{1}$ through $\delta s_{5}$, where the number corresponds to the surface number shown in Fig. 2. When treated individually, the inboard clamshell upper and lower surfaces will be denoted as $\delta s_{6 u}$ and $\delta s_{6 l}$, respectively. The outboard clamshell upper and lower surfaces will be similarly denoted $\delta s_{7 u}$ and $\delta s_{7 l}$. The winglet rudders are located on the wingtips and behave as normal rudders (affecting the directional axis only). The deflection of the winglet rudders is denoted $\delta r$. When an inner surface is coupled into an anti-symmetric pair with its counterpart on the opposite wing of the aircraft, the aileron-like combined deflection can be described by a single abstracted effector, $\delta a$, as shown in Eq. (1) . A similar definition for symmetric, elevator-like motion is described by Eq. (2).

$$
\begin{aligned}
& \delta a_{j}=\delta s_{j, l e f t}-\delta s_{j, r i g h t} \\
& \delta e_{j}=\delta s_{j, l e f t}+\delta s_{j, r i g h t}
\end{aligned}
$$




\section{Model Structure}

The multi-axis parameter estimation problem seeks to find the linear parameters that, when multiplied by their respective regressor functions, compute accurate values for axial force $\left(C_{A}\right)$, normal force $\left(C_{N}\right)$, side force $\left(C_{Y}\right)$, rolling moment $\left(C_{l}\right)$, pitching moment $\left(C_{m}\right)$, and yawing moment $\left(C_{n}\right)$. The assumed relationship between the regressor functions and the output force and moment coefficients is the model structure. The primary metrics for comparing the quality of the parameter estimates made by traditional methods versus multi-axis methods are the variance of the estimates, the Cramér-Rao lower bounds, and the time history reconstructions.

The multi-axis maneuvers are analyzed by solving the parameter estimation problem for both longitudinal and lateral-directional parameters simultaneously. The results of these analyses are then compared to parameter estimates obtained from single-axis maneuvers, which are analyzed by solving the equations for that axis only. The comparison then is between the single-axis (traditional) maneuvers which isolate motion as much as possible into ideally pure roll, pitch, or yaw, and multi-axis maneuvers which attempt to excite all axes at the same time.

For linear regression analysis, the general form is shown in Eq. (3).

$$
z=X \hat{\Theta},
$$

In Eq. (3), $z$ is the column array of force and moment coefficients, $X$ is the matrix of regressor functions (with individual model equations arranged in rows), and $\hat{\Theta}$ is the column array of parameters to be estimated. The force and moment coefficients are calculated from known or measured aircraft dynamics such as linear and rotational acceleration, instantaneous center of gravity, and moments of inertia. The regressor functions are known or constructed from aircraft state measurements, such as the angles of attack and sideslip, surface positions, and the instantaneous dynamic rates. Solving for the parameters yields Eq. (4):

$$
\hat{\Theta}=\left(X^{T} X\right)^{-1} X^{T} z
$$

For single-axis maneuvers, these equation sets are solved for in-axis derivatives only, in response to pure axis inputs. For example, a left surface and its corresponding right surface moving anti-symmetrically would produce (ideally) a pure roll input, and a response from the aircraft that should be dominated by roll-axis dynamics. An example equation for coefficient of rolling moment would be Eq. (5):

$$
C_{l}=C_{l o}+C_{l \beta} \cdot \beta+C_{l p} \cdot \frac{p b}{2 V}+C_{l r} \cdot \frac{r b}{2 V}+\left[C_{l, \text { surfaces }}\right]
$$

The first four terms of Eq. 5 are quite straightforward; the rolling moment should depend on some bareairframe damping term (the regressor function for which is simply 1), the angle of sideslip, and the roll and yaw rates of the aircraft. These aerodynamic stability and damping terms will be combined and referred to as $C_{l, a e r o}$ (and like manner for the directional coefficients) in the remainder of the paper; however, it should be noted that the four described components of $C_{l, \text { aero }}$ are solved for individually as their own regressor functions. An analogous example can be inferred for symmetric maneuvers acting purely on the pitch axis, or symmetric rudder maneuvers acting purely on the yaw axis.

In the case of single-surface, multi-axis excitations, the inputs are neither symmetric nor antisymmetric. Instead, a single surface on one side of the aircraft is subjected to a doublet input, and the asymmetry results in an excitation in the pitch, roll, and yaw axes simultaneously. Thus, the rolling moment coefficient equation becomes Eq. (6):

$$
C_{l, a e r o}=C_{l o}+C_{l \alpha} \cdot \alpha+C_{l \beta} \cdot \beta+C_{l p} \cdot \frac{p b}{2 V}+C_{l q} \cdot \frac{q c}{2 V}+C_{l r} \cdot \frac{r b}{2 V}
$$

In Eq. 6, longitudinal regressors for angle of attack and pitch rate are added to capture multi-axis dynamics. The corresponding $C_{l, \text { surfaces }}$ term would then consist of the the individual surface motions, as described by Eq. 7 .

$$
C_{l, \text { surfaces }}=\sum_{j} C_{l \delta s_{j}} \cdot d s_{j}
$$

The parameters are solved for using analogous equations for the remaining force and moment coefficients at each time frame of the data. The measured input data can be passed through the resulting equations (using 
the estimated parameters) to arrive at the equations of motion for the aircraft, which are then integrated to arrive at time history reconstructions of key state variables, such as angle of attack. These reconstructions can be compared to the measured, real-time histories to assess the accuracy of the parameter estimates. See Klein and Morelli $^{4}$ or Maine and Iliff ${ }^{7}$ for more detailed explanations of reconstructing the time histories using aircraft equations of motion, as well as the formulation of the force and moment coefficients used in this paper.

\section{E. Statistical Analyses}

Statistical analyses were performed on representative samples of flight data to determine the relative expected performance of the single-axis versus the multi-axis model. For the overall model equations, the tools used were the model coefficient of determination $\left(r^{2}\right)$ and the fit error $(s)$, defined in Eqs. (8) and (9), respectively. The average results for single- and multi-axis flight data taken at an angle of attack of 6 deg are shown in Table 1.

$$
\begin{aligned}
r^{2} & =\frac{\hat{\Theta}^{T} X^{T} z-N \bar{z}^{2}}{z^{T} z-N \bar{z}^{2}} \\
s & =\sqrt{\frac{\sum\left(z_{i}-\hat{y}_{i}\right)^{2}}{N-p}}
\end{aligned}
$$

\begin{tabular}{|c|c|c|c|}
\hline Model & Structure & $r^{2}$ & $s$ \\
\hline \hline$C_{l}$ & Single-axis & $92.10 \%$ & $27.80 \%$ \\
\hline & Multi-axis & $90.66 \%$ & $30.36 \%$ \\
\hline$C_{m}$ & Single-axis & $93.83 \%$ & $24.77 \%$ \\
\hline & Multi-axis & $92.69 \%$ & $26.97 \%$ \\
\hline$C_{n}$ & Single-axis & $90.44 \%$ & $30.79 \%$ \\
\hline & Multi-axis & $90.42 \%$ & $31.50 \%$ \\
\hline
\end{tabular}

Table 1. Average coefficient of determination $\left(r^{2}\right)$ and fit error $(s)$ for single-axis and multi-axis model structures, performed on three repetitions of representative flight data at $6 \mathrm{deg}$ trim angle of attack.

It should be noted that the results in Table 1 are for each model as applied to a supermaneuver constructed from sub-maneuvers that were designed for the model in question. In other words, the lateral-directional single-axis model was applied to a supermaneuver of anti-symmetric doublets, the longitudinal single-axis model was applied to a supermaneuver of symmetric doublets, and the multi-axis model was applied to a supermaneuver of asymmetric, single-surface doublets.

The coefficient of determination and the fit error are metrics that apply to the model equation as a whole. In addition, the $F_{0}$-statistic may be used to assess the relative statistical significance of of individual parameters in the regression fit. The $F_{0}$-statistic is described in Eq. (10), in which $s^{2}$ in this case is the parameter variance [not explicitly related to the fit error in Eq. (9)].

$$
F_{0}=\frac{\hat{\theta}_{j}^{2}}{s^{2}\left(\hat{\theta}_{j}\right)}
$$

Tables 2 and 3 show the F-ratio for rolling and pitching moment derivatives, respectively; they were calculated using the same method-specific samples of flight data as in the analyses of coefficient of determination and the fit error. In these tables, a higher value of the F-ratio connotes a higher level of significance to the regression fit.

For both the roll and the pitch axes, the multi-axis model is shown to exhibit F-ratios for the surface derivatives that are of the same order of magnitude as the single-axis model, though notably less in value. Overall, the significance values appear high; it should be noted that the manuevers in the flight data used for the analysis were designed specifically for these models.

All three statistical analyses suggest a priori that multi-axis models are likely to perform slightly worse than single-axis models with respect to model accuracy. 


\begin{tabular}{|c|c|c|}
\hline$C_{l}$ & $\delta a$ & $\delta s$ \\
\hline \hline Sfc 2 & $3.51 \cdot 10^{4}$ & $1.25 \cdot 10^{4}$ \\
\hline Sfc 3 & $4.58 \cdot 10^{4}$ & $1.69 \cdot 10^{4}$ \\
\hline Sfc 4 & $3.24 \cdot 10^{4}$ & $1.63 \cdot 10^{4}$ \\
\hline Sfc 5 & $4.89 \cdot 10^{4}$ & $1.74 \cdot 10^{4}$ \\
\hline
\end{tabular}

Table 2. Partial F-ratio for derivative of coefficient of rolling moment with respect to single-axis $(\delta a)$ and multi-axis $(\delta s)$ surface deflection.

\begin{tabular}{|c|c|c|}
\hline$C_{m}$ & $\delta e$ & $\delta s$ \\
\hline \hline Sfc 2 & $2.19 \cdot 10^{4}$ & $1.85 \cdot 10^{4}$ \\
\hline Sfc 3 & $2.28 \cdot 10^{4}$ & $1.83 \cdot 10^{4}$ \\
\hline Sfc 4 & $2.39 \cdot 10^{4}$ & $1.65 \cdot 10^{4}$ \\
\hline Sfc 5 & $2.41 \cdot 10^{4}$ & $1.63 \cdot 10^{4}$ \\
\hline
\end{tabular}

Table 3. Partial F-ratio for derivative of coefficient of pitching moment with respect to single-axis ( $\delta e)$ and multi-axis $(\delta s)$ surface deflection.

\section{Results and Discussion}

This section presents results of the comparison between the single-axis and multi-axis methods. Due to the proprietary nature of the performance data of the X-48B aircraft, all plots in this section are provided without quantification on the ordinate axis. The flight-data results are provided with error bars, which represent the Cramér-Rao lower bounds for the respective parameter estimate data points.

Many of the provided results depict trends for simulation and flight that do not agree with each other in slope or other characteristics. While the simulation and flight results will be loosely compared as a sanity check to ensure that the parameter estimation results for flight do not wildly deviate from expected values, it is not the objective of this paper to assess the accuracy of the Boeing nonlinear simulation or aerodynamic model with respect to flight. The objective is rather to compare the accuracy of the single-axis and multi-axis model on multiple HWB aircraft datasets.

The best measure of the effectiveness of a model lies in its ability to more accurately reconstruct observed state-variable time histories. In doing so, the best model should also perform with lower Cramér-Rao bounds than do other models for the same dataset. The models are here applied to two different datasets for the X-48B aircraft: simulation and flight. Any disparity between the two sets of results suggests that the aerodynamic model inadequately captures certain aerodynamics of the flight vehicle; it does not affect the comparison between the parameter estimation models.

Results are not shown for the innermost surface pair, surfaces 1, since the X-48B flight control laws do not permit a lateral roll input using this surface pair; they are designed for pitch control only.

\section{A. Aerodynamic Derivatives}

Representative aerodynamic derivatives for force and moment in the longitudinal and lateral-directional axes are shown in Figs. 4 and 5.

The multi-axis methods deliver estimates of aerodynamic derivatives that follow similar trends to the single-axis estimates. The multi-axis method, however, is shown to exhibit greater spread (corresponding to reduced consistency) and wider Cramér-Rao lower bounds (corresponding to increased uncertainty) than the single-axis method. Additionally, the average absolute percent difference in the individual parameter estimates can to be quite high, as shown in Table 4.

$C_{Y \beta}$ in particular appears to be estimated poorly. It should be noted, however, that in some cases the magnitudes of the derivatives are quite small. Thus, a small-magnitude difference in the estimates can lead to relatively high percentage differences. As another perspective on the error, for $C_{Y \beta}$, the relative difference between methods is approximately 6 times the span of the Cramér-Rao lower bound. The weakness in the modeling of $C_{Y \beta}$ by the multi-axis method is presumed to be related to the unique geometry of the X-48B 

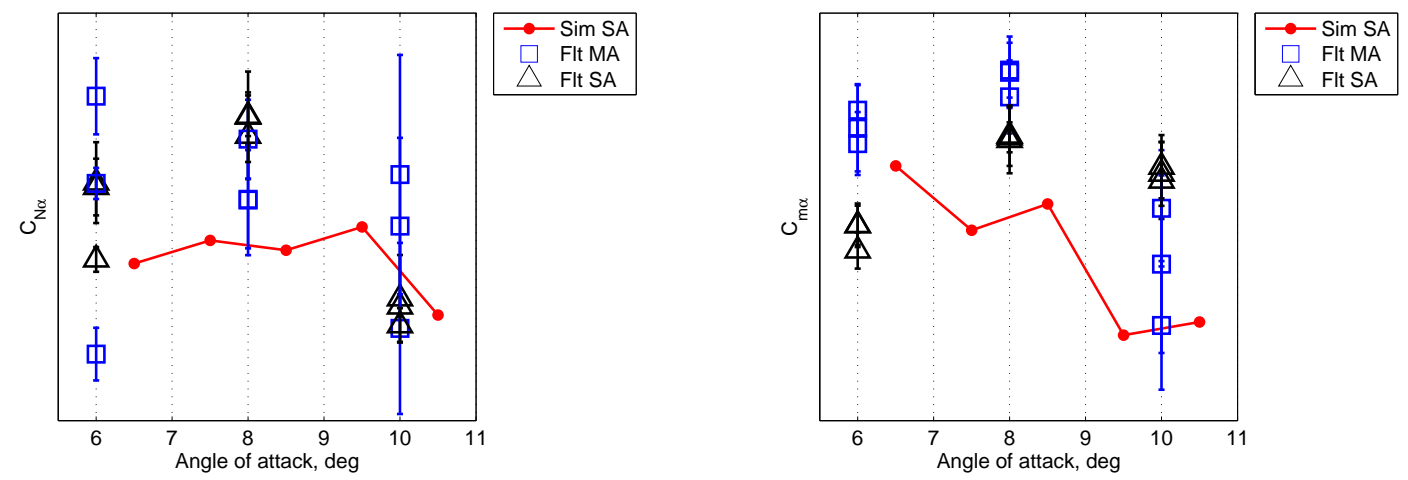

(a) Coefficient of normal force with respect to angle of at- (b) Coefficient of pitching moment with respect to angle of tack. attack.

Figure 4. Representative longitudinal aerodynamic derivatives for single-axis and multi-axis flight data.
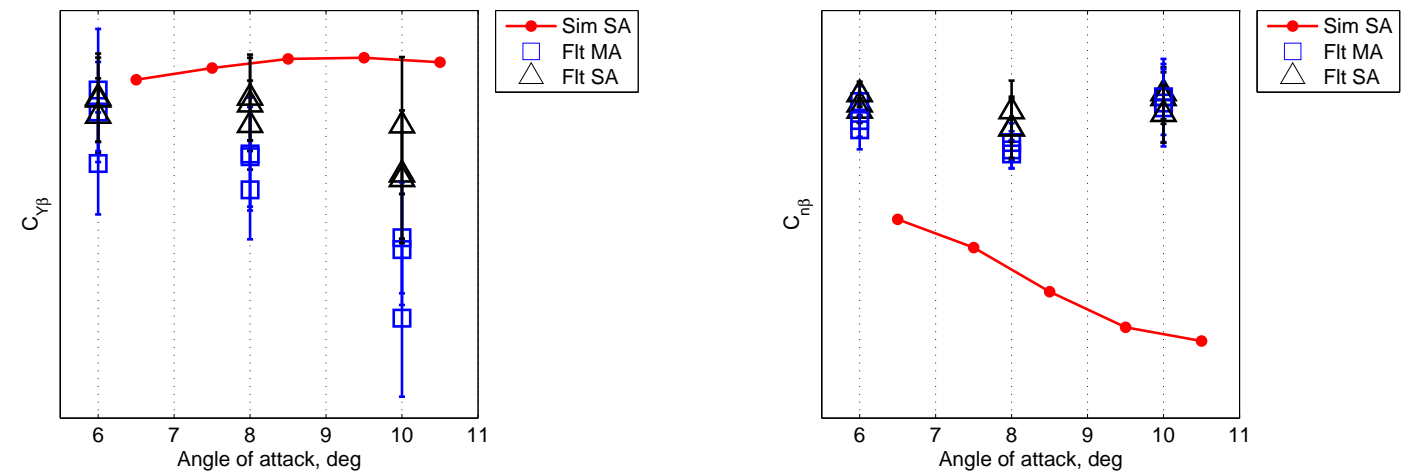

(a) Coefficient of side force with respect to angle of sideslip. (b) Coefficient of yawing moment with respect to angle of sideslip.

Figure 5. Representative lateral-directional aerodynamic derivatives for single-axis and multi-axis flight data.

\begin{tabular}{|c|c|}
\hline Derivative & Diff. \\
\hline \hline$C_{N \alpha}$ & $16.78 \%$ \\
\hline$C_{m \alpha}$ & $18.82 \%$ \\
\hline$C_{Y \beta}$ & $82.98 \%$ \\
\hline$C_{n \beta}$ & $13.51 \%$ \\
\hline$C_{l \beta}$ & $48.45 \%$ \\
\hline
\end{tabular}

Table 4. Average absolute percent difference between multi-axis and single-axis estimates of selected aerodynamic derivatives. 
aircraft, which is relatively flat in the XY plane; hence it has directional profile which does not change significantly with small changes in sideslip angle.

\section{B. Surface Derivatives}

Example parameter estimates for the pitch and roll effectiveness of the inner surfaces is shown in Figs. 6 through 9. In these figures, the parameters that were estimated using multi-axis maneuvers (designated "MA") were solved for using regression equations that included the full set of longitudinal and lateraldirectional terms. The single-axis maneuvers (designated "SA") were solved for using regression equations that isolated each axis, and used data sets from separate single-axis maneuvers.

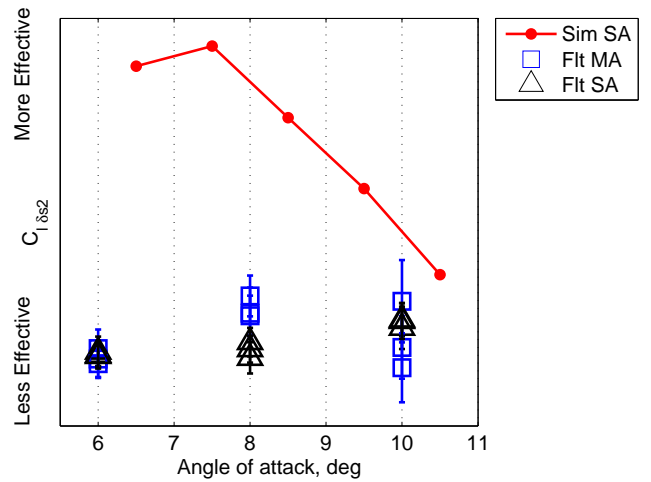

(a) Roll effectiveness.

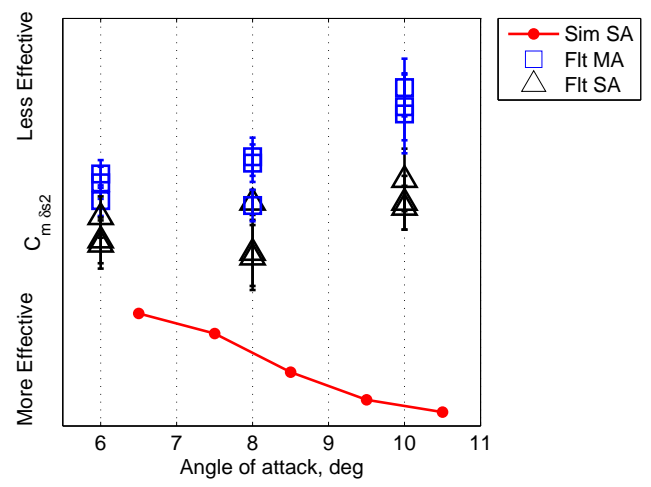

(b) Pitch effectiveness.

Figure 6. Estimates of surface 2 pitch and roll moment effectiveness.

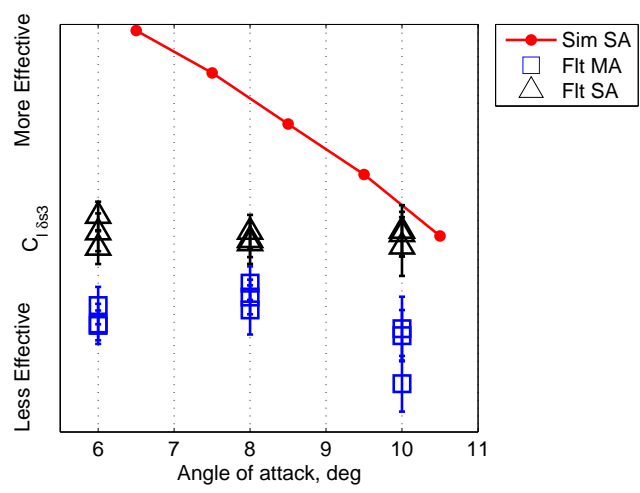

(a) Roll effectiveness.

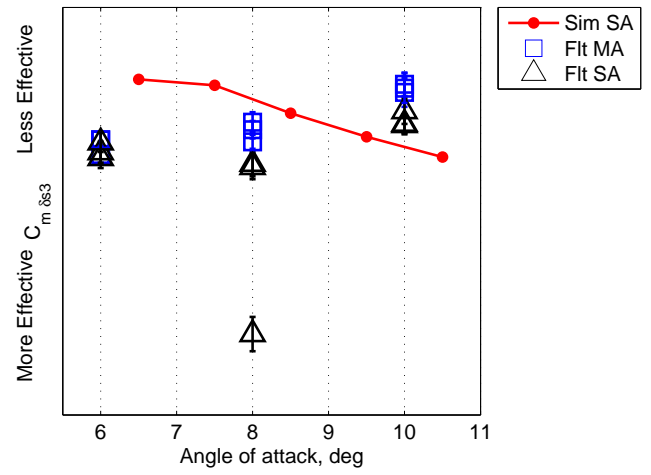

(b) Pitch effectiveness.

Figure 7. Estimates of surface 3 pitch and roll moment effectiveness.

Similar to the aerodynamic derivatives, the multi-axis methods deliver estimates of aerodynamic derivatives that follow similar trends to the single-axis estimates. The average absolute percent difference in the individual parameter estimates are shown in Table 5.

\begin{tabular}{|c|c|c|c|c|}
\hline Derivative & Diff., Sfc 2 & Diff., Sfc 3 & Diff., Sfc 4 & Diff., Sfc 5 \\
\hline \hline$C_{l \delta s}$ & $6.88 \%$ & $16.86 \%$ & $25.26 \%$ & $30.38 \%$ \\
\hline$C_{m \delta s}$ & $13.59 \%$ & $14.11 \%$ & $7.06 \%$ & $5.61 \%$ \\
\hline
\end{tabular}

Table 5. Average absolute percent difference between multi-axis and single-axis estimates of selected surface derivatives.

The pitch axis parameters are in general tightly clustered within each other's Cramér-Rao lower bounds for both single-axis and multi-axis models, though they appear to have larger Cramér-Rao lower bounds 


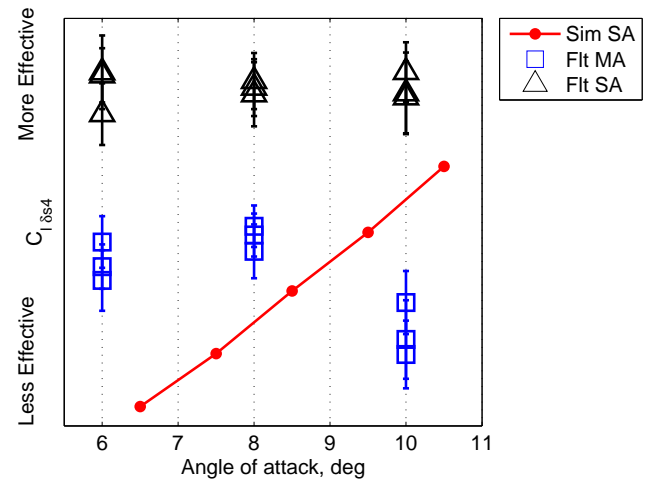

(a) Roll effectiveness.

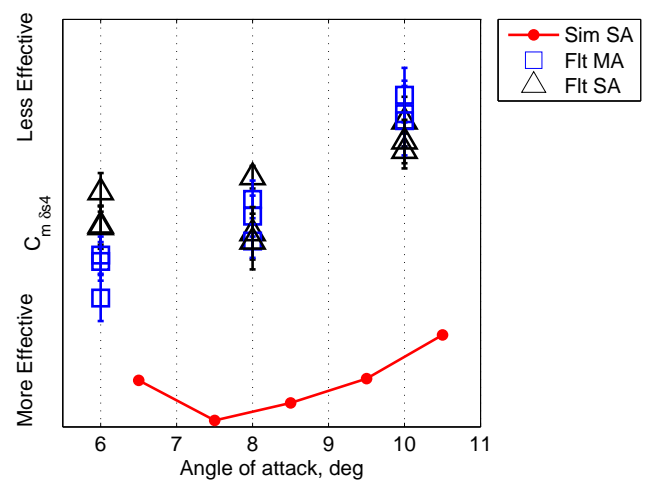

(b) Pitch effectiveness.

Figure 8. Estimates of surface 4 pitch and roll moment effectiveness.

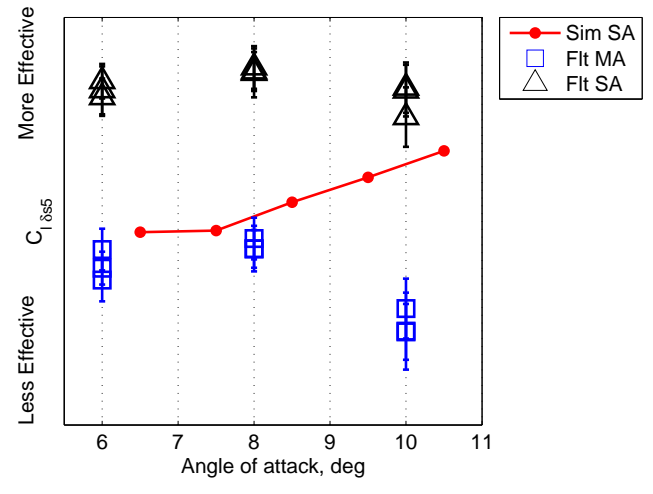

(a) Roll effectiveness.

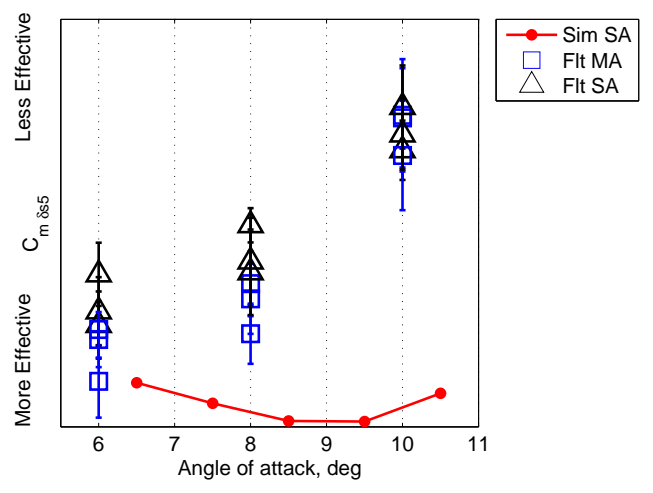

(b) Pitch effectiveness.

Figure 9. Estimates of surface 5 pitch and roll moment effectiveness. 
overall. The multi-axis estimates for rolling moment surface derivatives, however, agree less with the moreestablished single-axis method as one moves further outward on the wing. The multi-axis estimates for pitching moment surface derivatives in general appear to agree more with the single-axis methods as one moves further outward on the wing.

\section{Time History Reconstruction}

Time history reconstruction of state variables and moment coefficients is one of the most direct methods of assessing the quality of a parameter estimation model. When driven with the same inputs as simulation or flight, the model should be able to accurately reconstruct the observed outputs of the system under study. The calculated and observed moment equations for simulation and flight, respectively, were constructed using Eqs. (11) through (13).

$$
\begin{gathered}
\frac{\bar{q} S b}{I_{x x}} C_{l}=\dot{p}-\frac{I_{x z}}{I_{x x}} \dot{r}+\frac{I_{z z}-I_{y y}}{I_{x x}} q r-\frac{I_{x z}}{I_{x x}} q p \\
\frac{\bar{q} S c}{I_{y y}} C_{m}=\dot{q}+\frac{I_{x x}-I_{z z}}{I_{y y}} p r+\frac{I_{x z}}{I_{y y}}\left(p^{2}-r^{2}\right)-\frac{I_{p}}{I_{y y}} \Omega_{p} r \\
\frac{\bar{q} S b}{I_{z z}} C_{n}=\dot{r}-\frac{I_{x z}}{I_{z z}} \dot{p}+\frac{I_{y y}-I_{x x}}{I_{z z}} p q+\frac{I_{x z}}{I_{z z}} q r+\frac{I_{p}}{I_{z z}} \Omega_{p} q
\end{gathered}
$$

The parameter estimates from each model were used to reconstruct estimates of the above experimental values using the parameter equations as in Eqs. 5 and 6. The time histories of these were then compared to the calculated (simulation) or observed (flight) time histories. The force and moment coefficients can then be integrated using the equations of motion to arrive at the aircraft state variables, such as wind angles and rotational rates.

\section{A. Simulation}

Example time history reconstructions of the rolling, pitching, and yawing moments for simulation data are shown in Figs. 10 through 12. These figures represent the same slice from a supermaneuver assembled for 9.5 deg angle of attack.

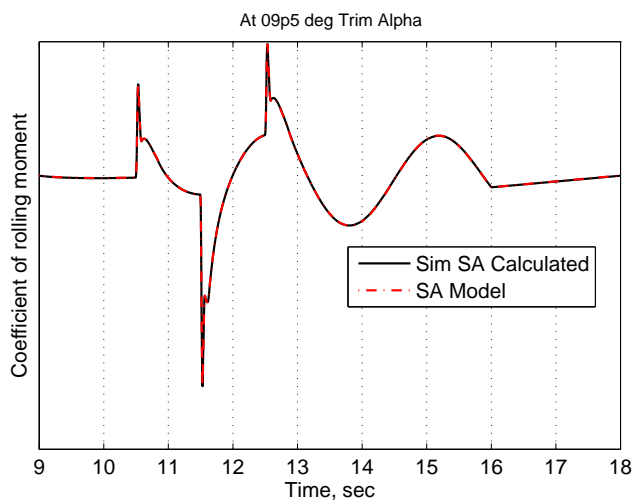

(a) Single-axis model.

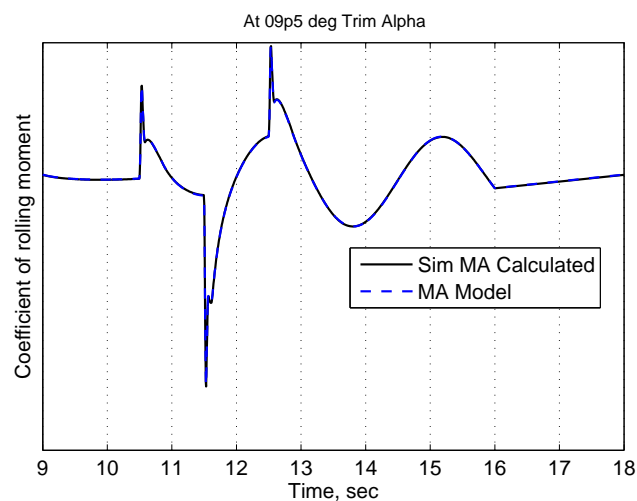

(b) Multi-axis model.

Figure 10. Coefficient of rolling moment for single-axis and multi-axis models, reconstructed from parameter estimates on simulation data at 9.5 deg trim angle of attack.

The reconstructions of the coefficients on simulation data verify that the models behave as expected in a nearly ideal experimental context: open-loop, noiseless, and perfectly controlled. The single-axis and multi-axis models reconstruct the moment coefficients nearly perfectly. 


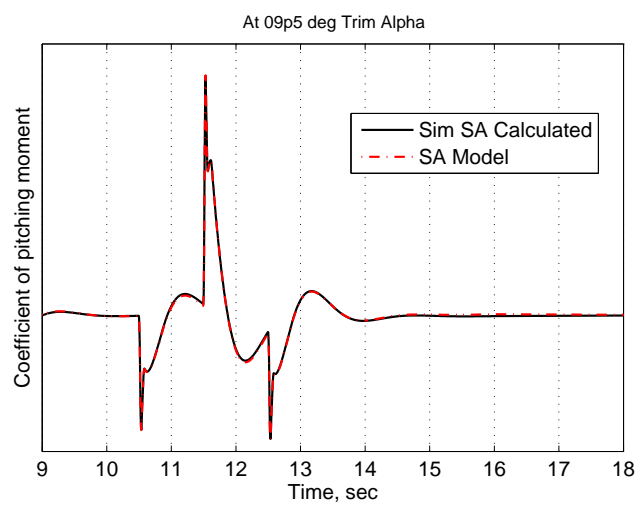

(a) Single-axis model.

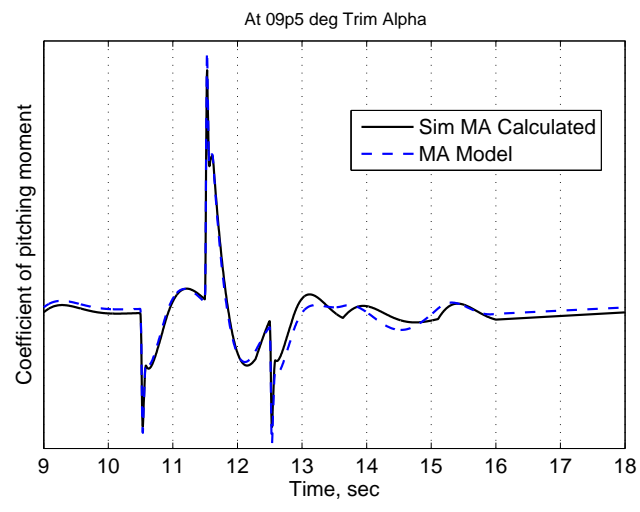

(b) Multi-axis model.

Figure 11. Coefficient of pitching moment for single-axis and multi-axis models, reconstructed from parameter estimates on simulation data at $9.5 \mathrm{deg}$ trim angle of attack.

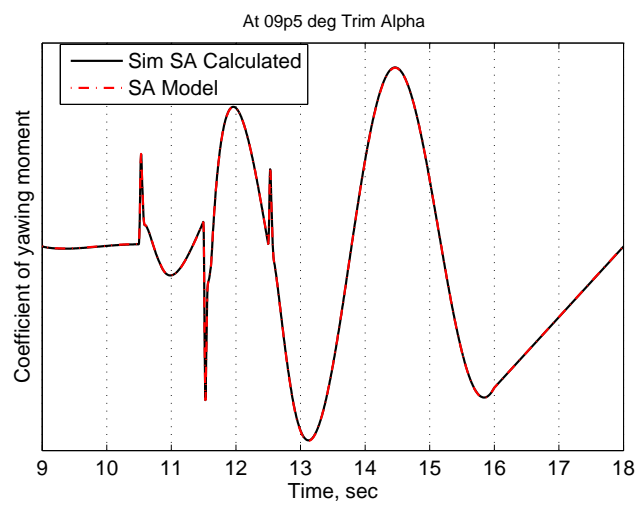

(a) Single-axis model.

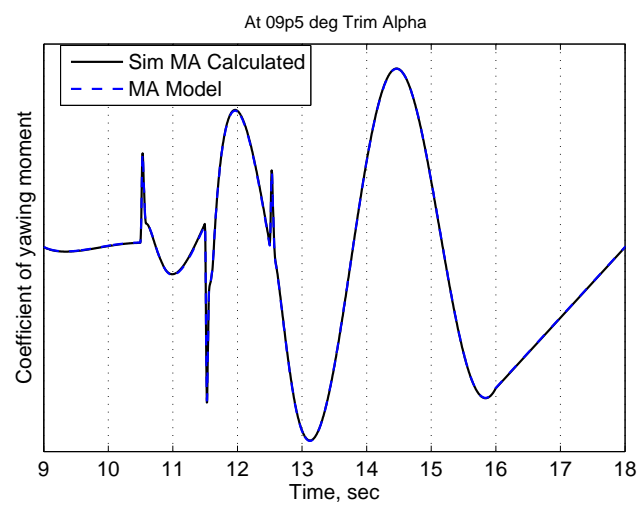

(b) Multi-axis model.

Figure 12. Coefficient of yawing moment for single-axis and multi-axis models, reconstructed from parameter estimates on simulation data at $9.5 \mathrm{deg}$ trim angle of attack. 


\section{B. Flight}

Reconstructions for the roll, pitch, and yaw axis moment coefficients for flight data are shown in Figs. 13 through 15. As with the simulation maneuvers, both the single-axis and multi-axis maneuvers were performed separately. Unlike in simulation, however, the environment is not as controlled as in flight, and there are differences in the two sets due to expected variations in the nature of the test points. Thus for the flight coefficient reconstructions, the single-axis results are plotted separately from the multi-axis results in order to show legitimate comparisons. All reconstructions are shown for the same surface deflection as a representative case: $\delta s 2$ versus $\delta a 2$ for the lateral-directional coefficients, and $\delta s 2$ versus $\delta e 2$ for the longitudinal coefficients.

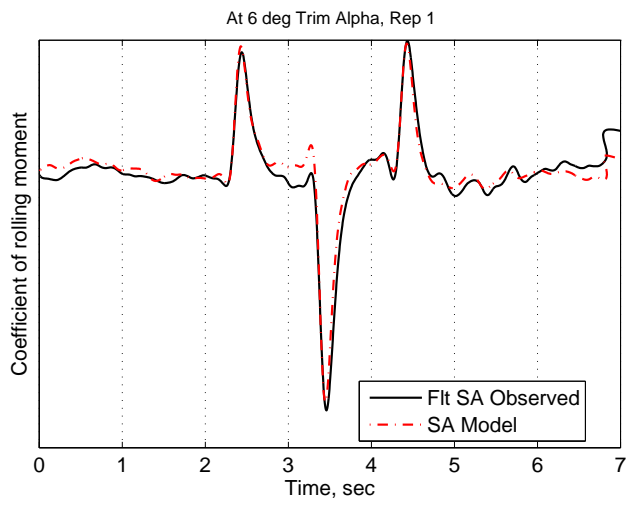

(a) Single axis model.

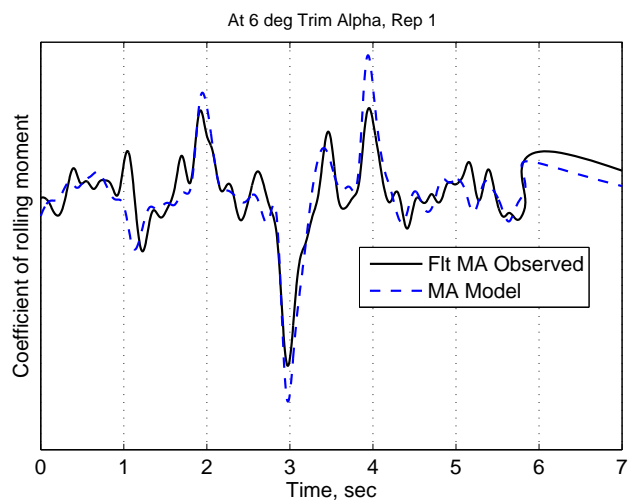

(b) Multi-axis model.

Figure 13. Coefficient of rolling moment for single-axis and multi-axis models, reconstructed from parameter estimates on flight data at $6 \mathrm{deg}$ trim angle of attack.

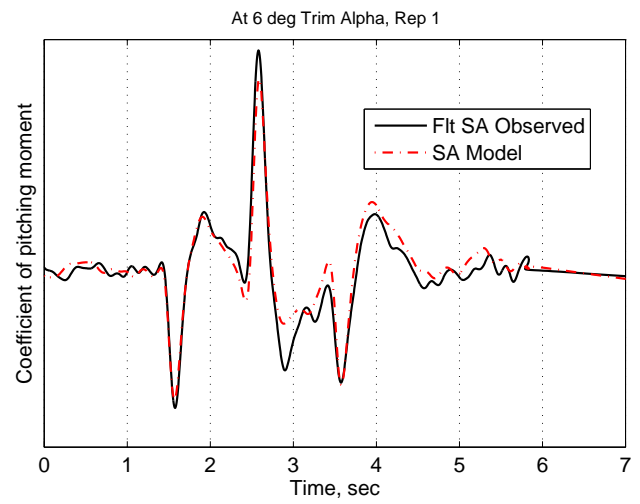

(a) Single axis model.

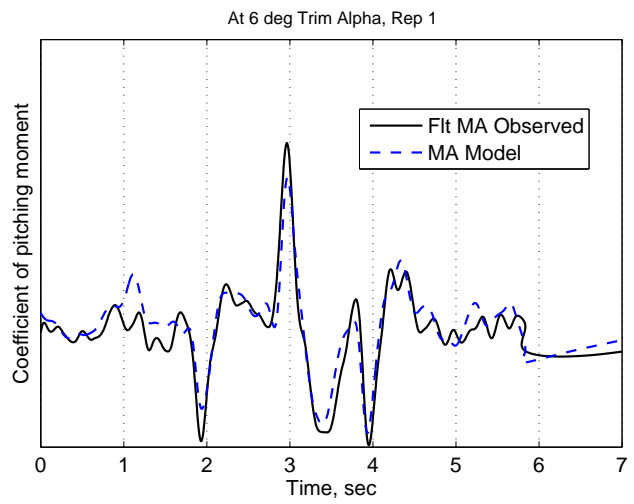

(b) Multi-axis model.

Figure 14. Coefficient of pitching moment for single-axis and multi-axis models, reconstructed from parameter estimates on flight data at $6 \mathrm{deg}$ trim angle of attack.

The flight data reconstructions show the baseline, single-axis model performing very well, as expected. The multi-axis model exhibits noticeably degraded accuracy in the reconstruction, but the trends in the observed time histories are in fact captured quite well. Overall, it appears that the multi-axis model does perform close to the single-axis model in terms of moment coefficient reconstruction, achieving fit errors of roughly $1-5 \%$.

Representative plots of the reconstructions of aircraft wind angles and rotational rates are shown in Figs. 16 through 19.

The calculated angles of attack for both single-axis and multi-axis models, in Fig. 16, are passable but weak. The multi-axis model does appear to be slightly worse, but the difference is not significant. The calculated angles of sideslip exhibit a better comparison. In Fig. 17, the single-axis model is seen to very accurately reconstruct the observed angle of sideslip; the multi-axis model performs less well, but still 


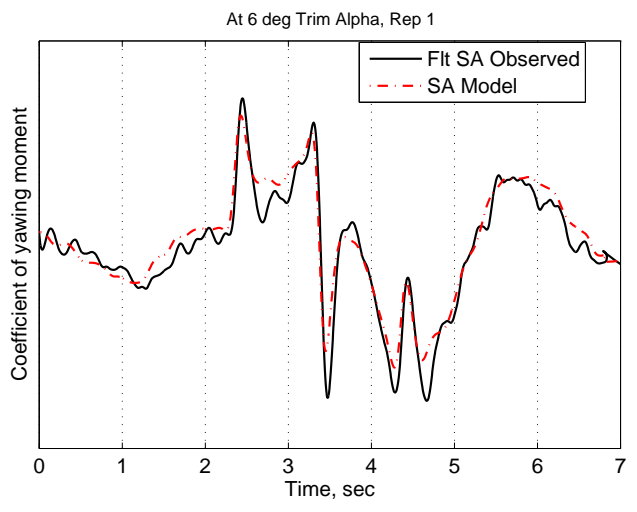

(a) Single axis model.

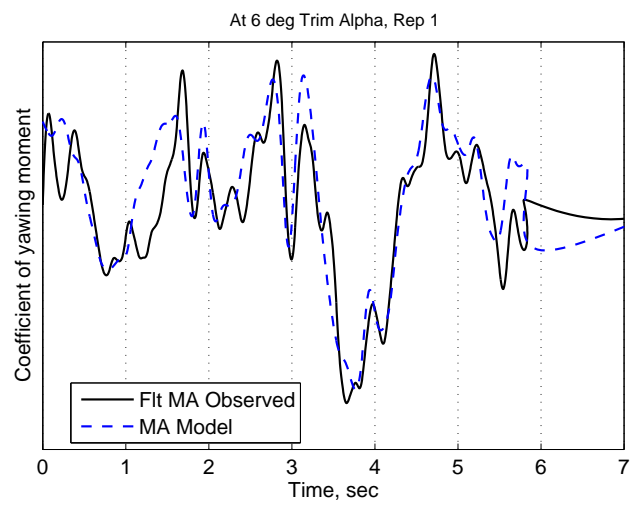

(b) Multi-axis model.

Figure 15. Coefficient of yawing moment for single-axis and multi-axis models, reconstructed from parameter estimates on flight data at 6 deg trim angle of attack.

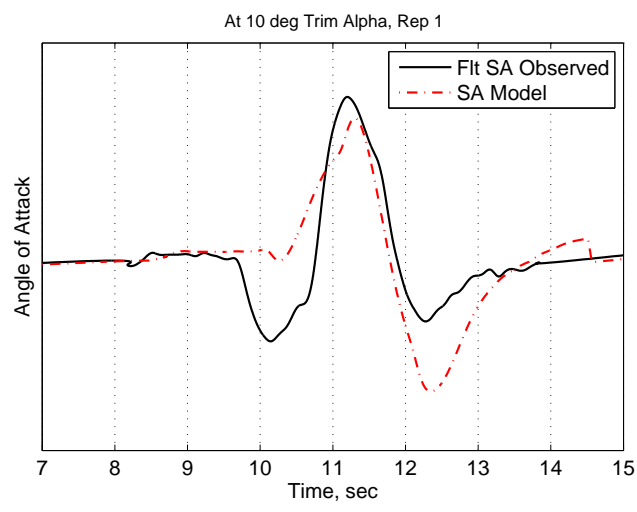

(a) Single axis model, 10 deg trim angle of attack.

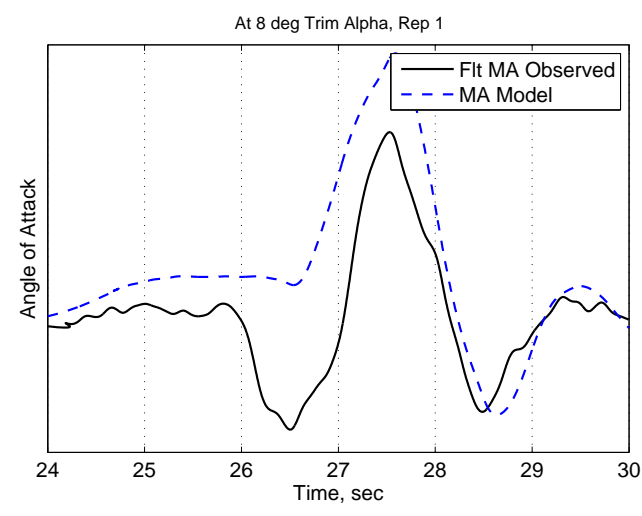

(b) Multi-axis model, $8 \mathrm{deg}$ trim angle of attack.

Figure 16. Angle of attack for single-axis and multi-axis models, integrated from force and moment coefficient reconstructions on flight data.

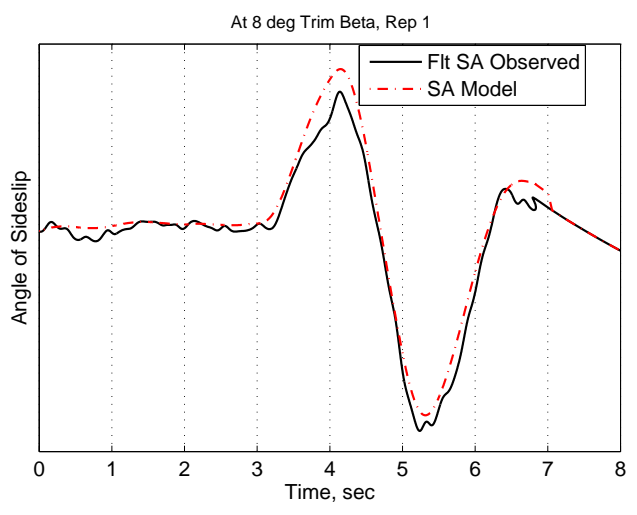

(a) Single axis model, 8 deg trim angle of attack.

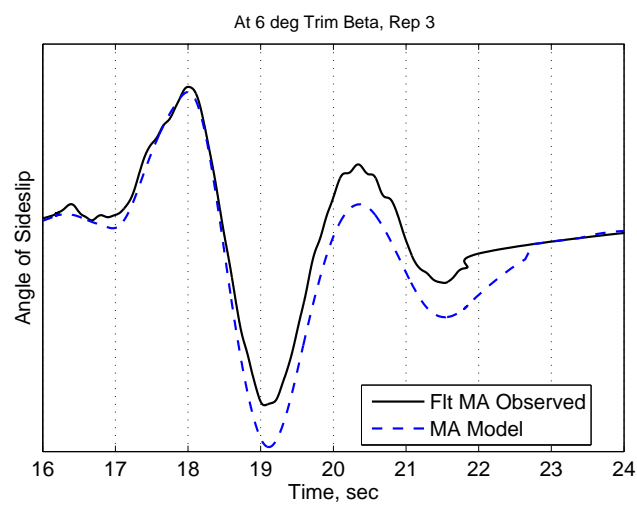

(b) Multi-axis model, 6 deg trim angle of attack.

Figure 17. Angle of sideslip for single-axis and multi-axis models, integrated from force and moment coefficient reconstructions on flight data. 


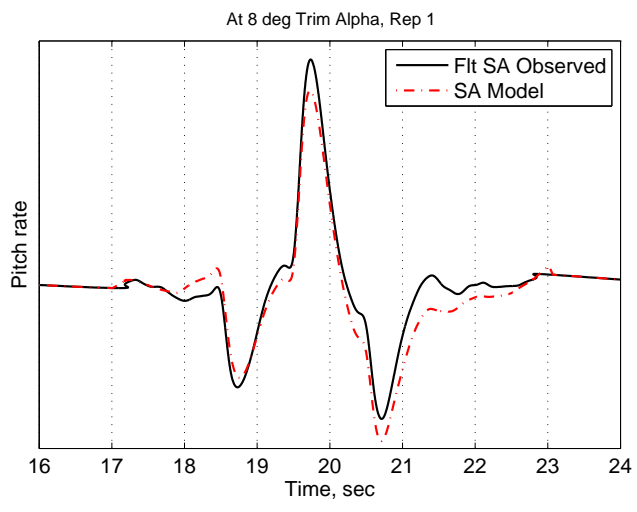

(a) Single axis model, 8 deg trim angle of attack.

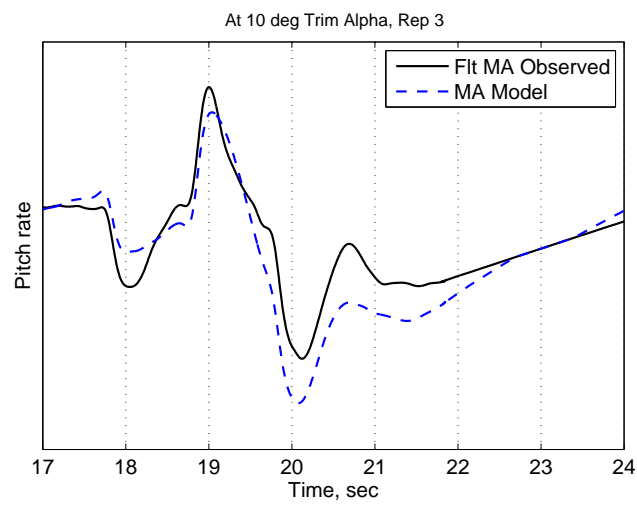

(b) Multi-axis model, 10 deg trim angle of attack.

Figure 18. Pitch rate for single-axis and multi-axis models, integrated from force and moment coefficient reconstructions on flight data.

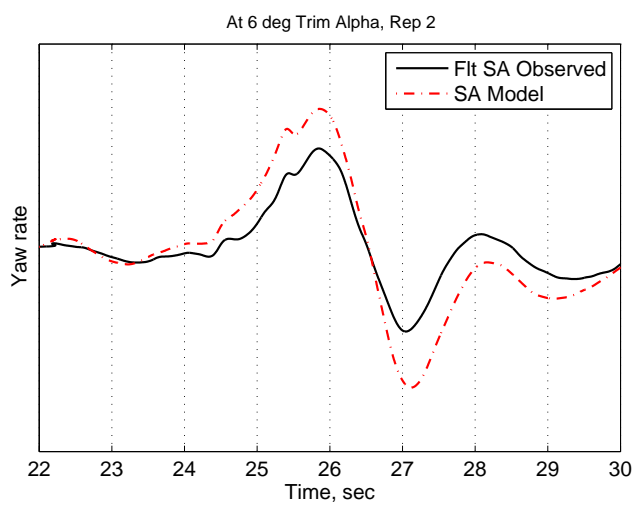

(a) Single axis model, 6 deg trim angle of attack.

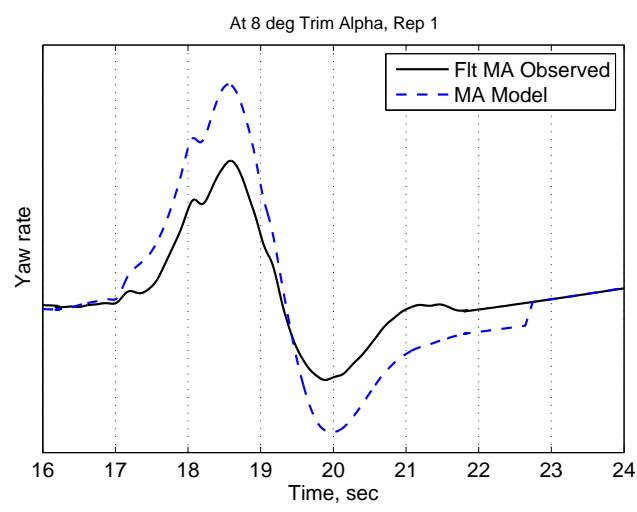

(b) Multi-axis model, $8 \mathrm{deg}$ trim angle of attack.

Figure 19. Yaw rate for single-axis and multi-axis models, integrated from force and moment coefficient reconstructions on flight data. 
manages to capture the important features of the motion. Similar conclusions may be drawn from observation of the pitch rate reconstructions in Fig. 18. No significant differences in the quality of reconstruction, however, may be readily drawn from the plots of calculated yaw rate in Fig. 19.

\section{Concluding Remarks}

Multi-axis methods provided parameter estimates that were generally similar to single-axis methods in terms of trends, but individual parameters were often quite different by a typical average absolute error of roughly 15-20\%, with decreased statistical significance. Regression on flight data indicated that a multi-axis model exhibited an increase in overall fit error of roughly $1-5 \%$ for the linear regression estimates of the force and moment coefficients with respect to a single-axis model. These errors were calculated as applied to flight data designed for each method. Multi-axis reconstructions of aircraft state variables were shown to be noticeably, but not significantly, poorer than single-axis reconstructions of the same wind angles and rotational rates.

Since the multi-axis maneuvers captured similar parameter estimates as both longitudinal and lateraldirectional maneuvers combined, the number of flight-test points for the inner surfaces could have theoretically been reduced by $50 \%$. This reduction, however, came at the cost of moderately degraded accuracy in individual parameter estimates and slightly degraded accuracy in overall model fit error. Additionally, removal of half of the required test points will not necessarily reduce the overall flight time by the same amount, as the overhead involved in operational considerations (including getting on-condition, fueling, turns to stay within boundaries, et cetera) does not scale linearly and is usually highly dependent on the design of the test matrix.

The inner surfaces of the X-48B Blended Wing Body, on which this study was focused, are relatively straightforward in terms of function and motion; application of multi-axis techniques to the more complex outer surface clamshell motion could result in different performance for multi-axis methods. Future parameter estimation research at the NASA Dryden Flight Research Center (Edwards, California) may delve into these problems of interest for hybrid-wing-body type aircraft.

\section{References}

\footnotetext{
${ }^{1}$ Taylor, B.R. and Ratnayake, N.A., "Simulation and Flight Evaluation of a Parameter Estimation Input Design Method for Hybrid-Wing-Body Aircraft," AIAA 2010-7949, 2010.

${ }^{2}$ Ratnayake, N.A. and Waggoner, E.R., and Taylor, B.R., "Lateral-Directional Parameter Estimation on the X-48B Using an Abstracted, Multi-Objective Effector Model," AIAA-2011-, 2011.

${ }^{3}$ Maine, R. E. and Iliff, K. W., "Identification of Dynamic Systems-Theory and Formulation," NASA RP-1138, 1985.

${ }^{4}$ Klein, V. and Morelli, E.A., Aircraft System Identification: Theory and Practice, AIAA Education Series, 2006.

${ }^{5}$ Steers, S. T. and Iliff, K. W., "Effects of Time-Shifted Data on Flight-Determined Stability and Control Derivatives," NASA TN D-7830, 1975.

${ }^{6}$ Iliff, K. W. and Maine, R. E. and Montgomery, T. D., "Important Factors in the Maximum Likelihood Analysis of Flight Test Maneuvers," NASA TP-1459, 1979.

${ }^{7}$ Maine, R.E. and Iliff, K.W., "Application of Parameter Estimation to Aircraft Stability and Control - The Output Error Approach," NASA RP-1168, 1986.

${ }^{8}$ Trujillo, B. M., "Determination of Lift and Drag Characteristics of Space Shuttle Orbiter Using Maximum Likelihood Estimation Technique," AIAA 86-2225, 1986.

${ }^{9}$ Neto, N.S.B. and Hemerly, E.M. and Goes, L.C.S., "Aircraft Parameter Estimation Experiment Design Considering Measurement Colored Residuals," Journal of Aircraft, Vol. 46, No. 6, 2009, pp. $1857-1865$.

${ }^{10}$ Morelli, E. A., "F-18 High Alpha Research Vehicle (HARV) Parameter Identification Flight Test Maneuvers for Optimal Input Design Validation and Lateral Control Effectiveness," NASA CR-198248, 1995.

${ }^{11}$ Morelli, E.A., "Flight Test Validation of Optimal Input Design and Comparison to Conventional Inputs," AIAA-97-3711, 1997.

${ }^{12}$ Morelli, E. A., "Flight-Test Experiment Design for Characterizing Stability and Control of Hypersonic Vehicles," Journal of Guidance, Control, and Dynamics, Vol. 32, No. 3, 2009, pp. 949-959.

${ }^{13}$ Morelli, E. A., "Multiple Input Design for Real-Time Parameter Estimation in the Frequency Domain," 13th IFAC Symposium on System Identification, 2003.
} 
${ }^{14}$ Morelli, E. A., "Practical Input Optimization for Aircraft Parameter Estimation Experiments," NASA CR$191462,1993$.

${ }^{15}$ Morelli, E. A. and Klein, V., "Optimal Input Design for Aircraft Parameter Estimation using Dynamic Programming Principles," AIAA 90-2801-CP, 1990.

${ }^{16}$ Maine, R.E. and Iliff, K.W., "Theory and Practice of Estimating the Accuracy of Dynamic Flight-Determined Coefficients," NASA RP-1077, 1981. 
Multi-Axis Identifiability Using Single-Surface Parameter Estimation Maneuvers on the X-48B

\title{
Blended Wing Body
}

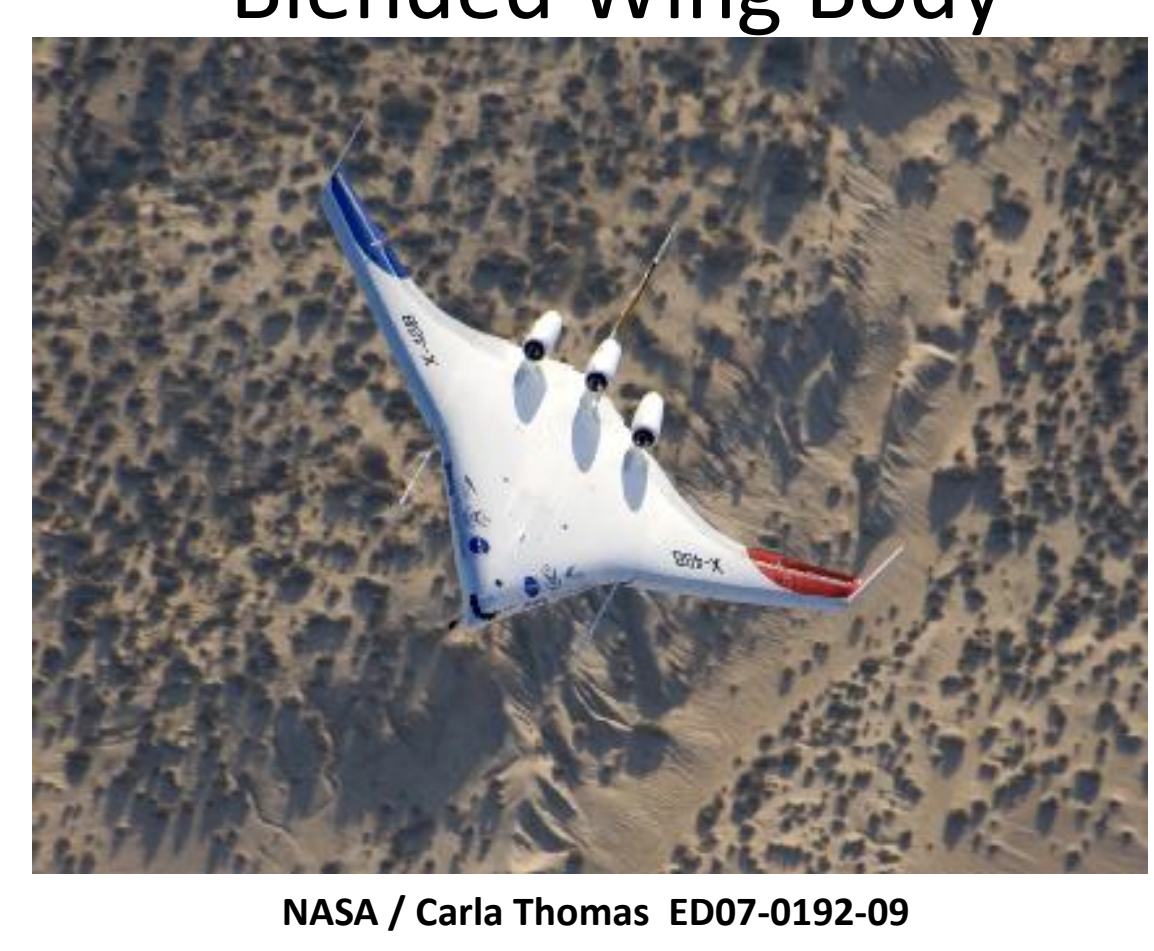

\author{
Nalin A. Ratnayake \\ Ed T. Koshimoto \\ Brian R. Taylor
}

NASA Dryden Flight Research Center 


\section{Problem Statement}

- Hybrid-Wing Body aircraft such as the Boeing X-48B BWB are potentially the future of highly efficient air transport

- New systems will possibly require new ways of approaching the system identification problem

- Math is the same, but...

- New configurations $=$ new issues

- Identifiability!

- Many co-planar surfaces

- Complex outer surface motion (clamshell)

- This paper: tradeoff on accuracy when using asymmetric maneuvers to attempt to

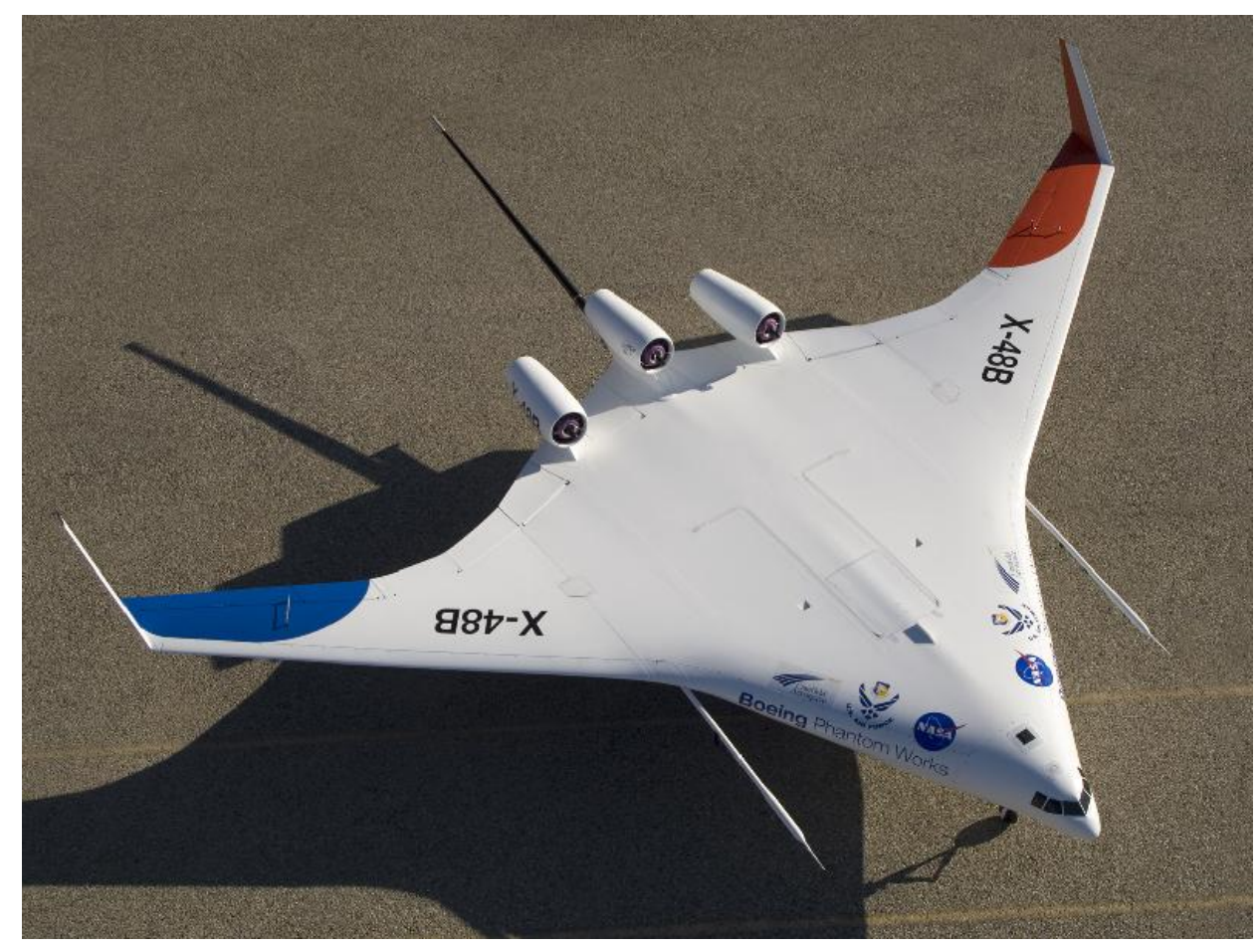

NASA / Tony Landis (ED06-0198-37) estimate multiple axes at once (time savings)

Multi-axis versus single-axis... how to they compare? 


\section{Surface Definitions}

- Surfaces 1: elevator motion

- Surfaces 2-5: elevon motion

- Surfaces 6/8 and 7/9: clamshell motion

- Aileron-like for roll

- Split motion for drag rudder mode

- Winglet rudders
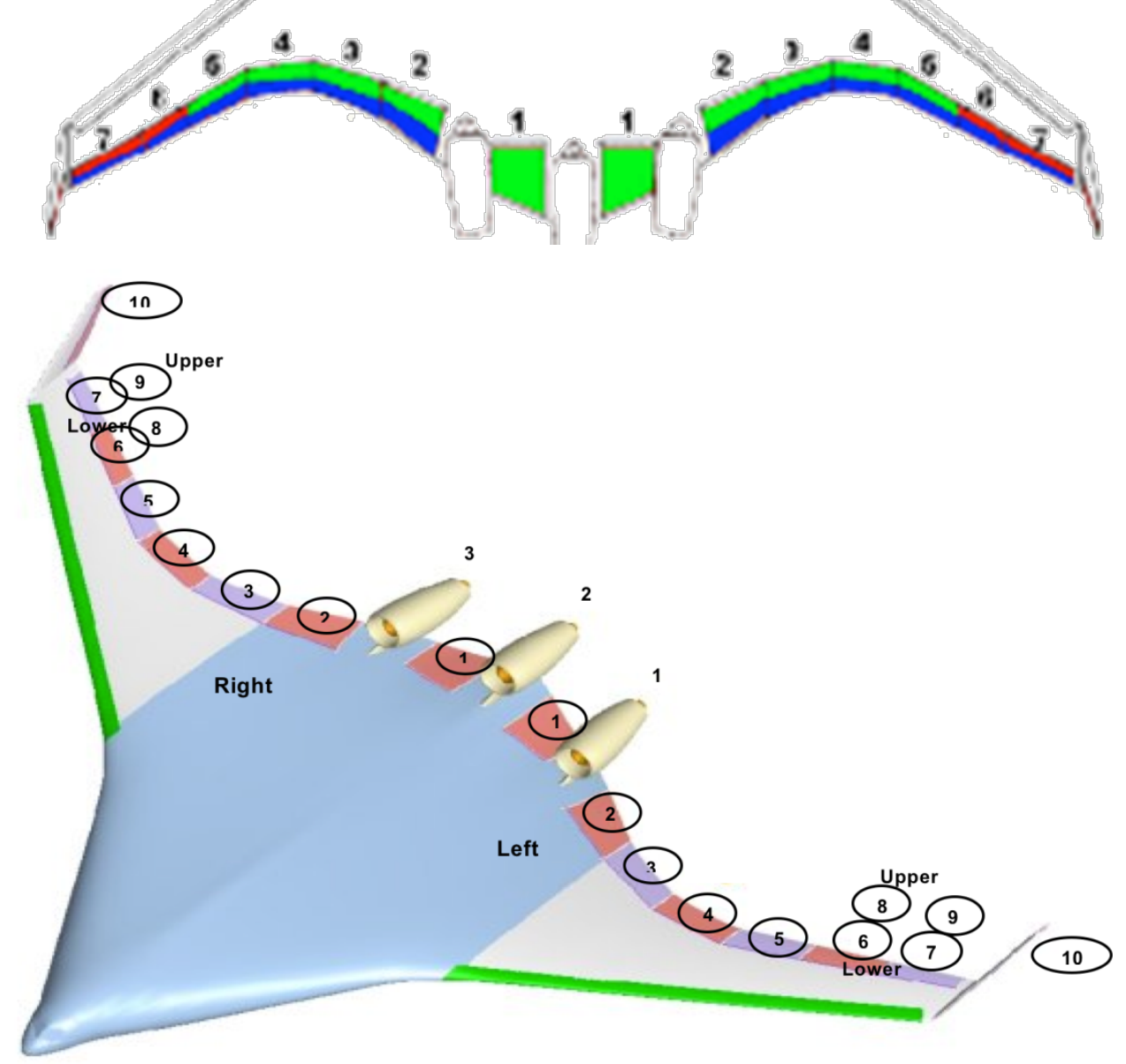


\section{Supermaneuver}

- Individual doublets are spliced together into a single data file for combined analysis

- 2 sec time frame skip to reset integrators

- Single-axis (pitch): symmetric motion

- Single-axis (roll): antisymmetric motion

- Multi-axis (pitch and roll):

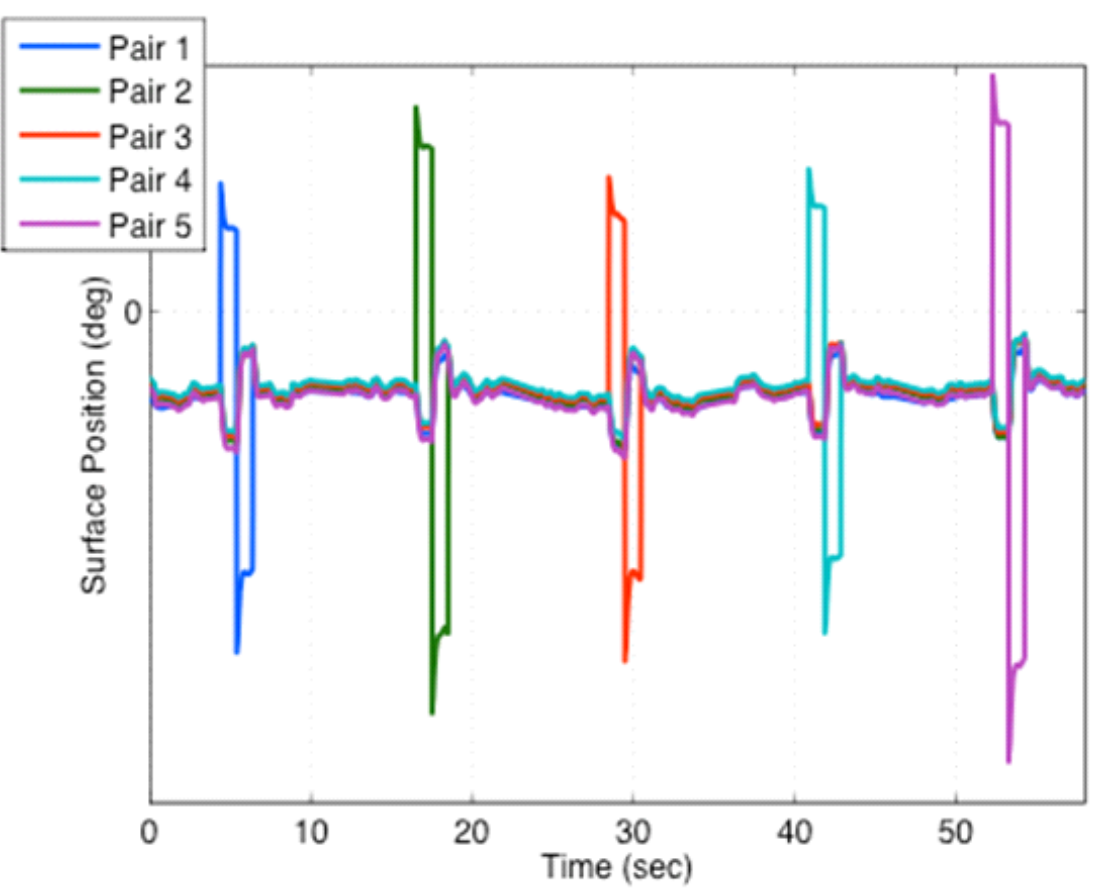
asymmetric motion 


\section{Data Sources}

- Simulation

- Boeing nonlinear sim v4.3, VMS v4.3.1, aero model 20100527, implemented in Simulink

- PID/OBES and control allocator modified by DFRC for maneuver definition and forcing open-loop

- Flight

- Flights 65, 66, 67, 85, 89

- Flight data filtered though $3^{\text {rd }}$ Order Butterworth filter, $5 \mathrm{~Hz}$ corner frequency

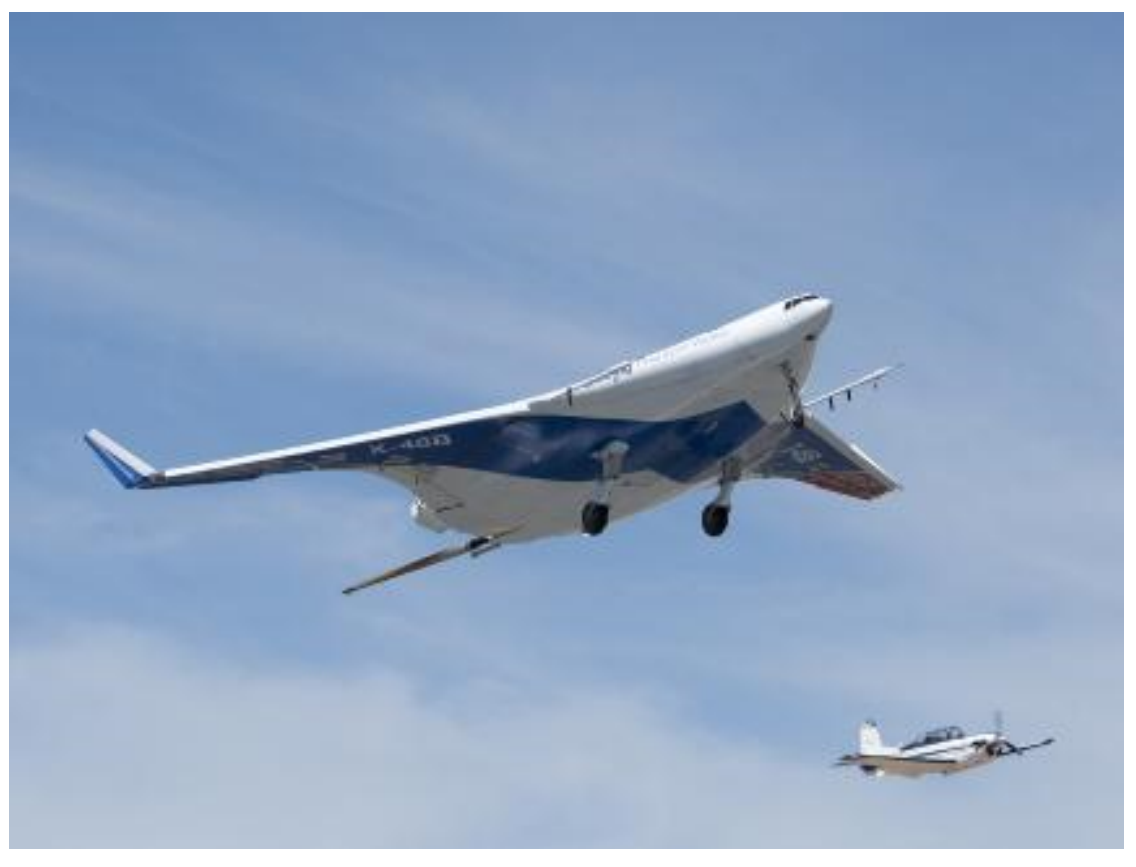

NASA / Tony Landis (ED10-0056-32) 


\section{Linear Regression for PID}

Outputs $=$ Regressors $*$ Parameters

- Model form is assumed to be linear (though regressors may be nonlinear)

- Use statistical tools to $C_{l}=C_{l o}+C_{l \beta} \cdot \beta+C_{l p} \cdot \frac{p b}{2 V}+C_{l r} \cdot \frac{r b}{2 V}+\left[C_{l, s u r f a c e s}\right]$ assess model quality and parameter significance

$$
r^{2}=\frac{\hat{\Theta}^{T} X^{T} z-N \bar{z}^{2}}{z^{T} z-N \bar{z}^{2}}
$$

- Estimate uncertainty from Cramér-Rao bounds

$$
\begin{gathered}
s=\sqrt{\frac{\sum\left(z_{i}-\hat{y}_{i}\right)^{2}}{N-p}} \\
F_{0}=\frac{\hat{\theta}_{j}^{2}}{s^{2}\left(\hat{\theta}_{j}\right)}
\end{gathered}
$$




\section{Statistical Analysis}

- Analysis of coefficient of determination, fit-error, and significance suggest a priori that the multiaxis model is likely to perform worse than the single-axis model

- Confirms intuition

\begin{tabular}{|c|c|c|c|}
\hline Model & Structure & $r^{2}$ & $s$ \\
\hline \hline$C_{l}$ & Single-axis & $92.10 \%$ & $27.80 \%$ \\
\hline & Multi-axis & $90.66 \%$ & $30.36 \%$ \\
\hline$C_{m}$ & Single-axis & $93.83 \%$ & $24.77 \%$ \\
\hline & Multi-axis & $92.69 \%$ & $26.97 \%$ \\
\hline$C_{n}$ & Single-axis & $90.44 \%$ & $30.79 \%$ \\
\hline & Multi-axis & $90.42 \%$ & $31.50 \%$ \\
\hline
\end{tabular}

\begin{tabular}{|c|c|c|}
\hline$C_{l}$ & $\delta a$ & $\delta s$ \\
\hline \hline Sfc 2 & $3.51 \cdot 10^{4}$ & $1.25 \cdot 10^{4}$ \\
\hline Sfc 3 & $4.58 \cdot 10^{4}$ & $1.69 \cdot 10^{4}$ \\
\hline Sfc 4 & $3.24 \cdot 10^{4}$ & $1.63 \cdot 10^{4}$ \\
\hline Sfc 5 & $4.89 \cdot 10^{4}$ & $1.74 \cdot 10^{4}$ \\
\hline
\end{tabular}

\begin{tabular}{|c|c|c|}
\hline$C_{m}$ & $\delta e$ & $\delta s$ \\
\hline \hline Sfc 2 & $2.19 \cdot 10^{4}$ & $1.85 \cdot 10^{4}$ \\
\hline Sfc 3 & $2.28 \cdot 10^{4}$ & $1.83 \cdot 10^{4}$ \\
\hline Sfc 4 & $2.39 \cdot 10^{4}$ & $1.65 \cdot 10^{4}$ \\
\hline Sfc 5 & $2.41 \cdot 10^{4}$ & $1.63 \cdot 10^{4}$ \\
\hline
\end{tabular}




\section{Selected Aerodynamic Derivs}
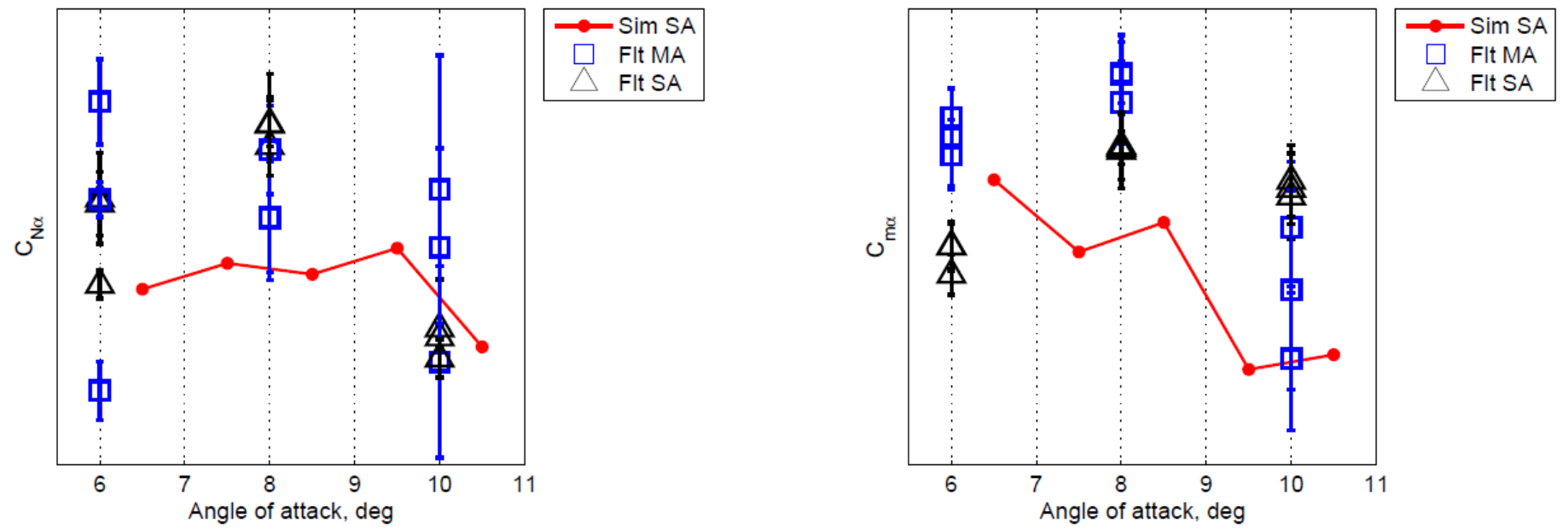

\begin{tabular}{|c|c|}
\hline Derivative & Diff. \\
\hline \hline$C_{N \alpha}$ & $16.78 \%$ \\
\hline$C_{m \alpha}$ & $18.82 \%$ \\
\hline$C_{Y \beta}$ & $82.98 \%$ \\
\hline$C_{n \beta}$ & $13.51 \%$ \\
\hline$C_{l \beta}$ & $48.45 \%$ \\
\hline
\end{tabular}
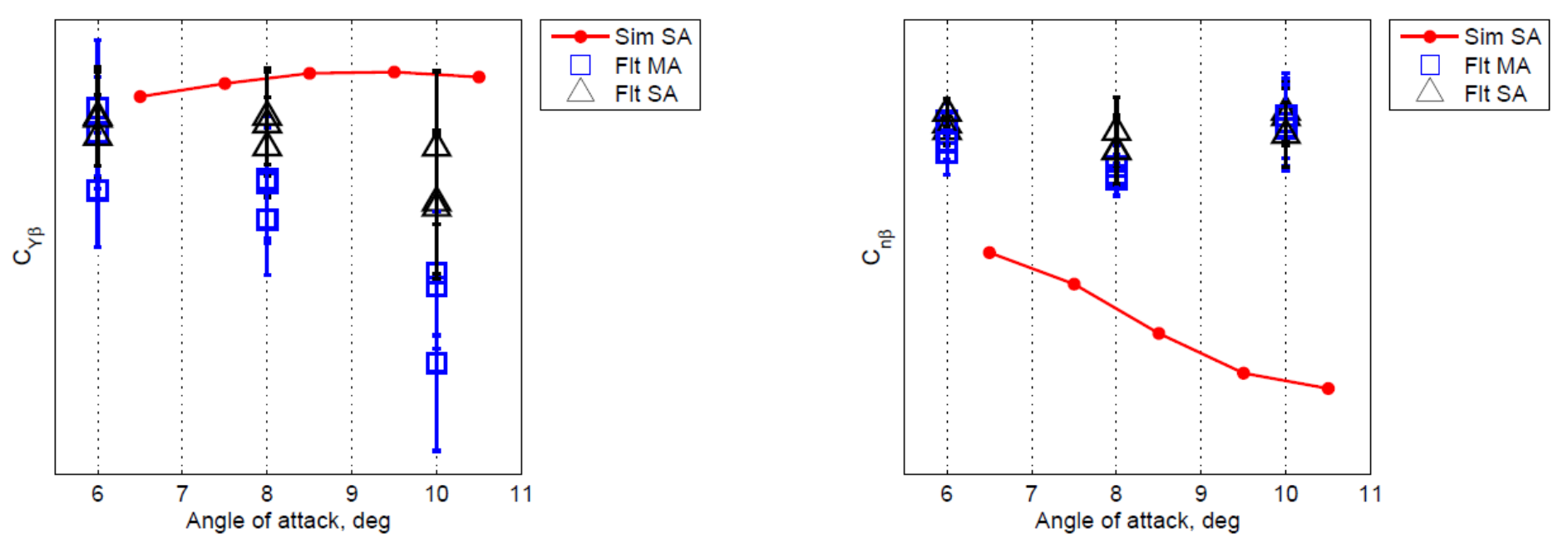


\section{Roll/pitch derivs. (Sfcs 2,3)}
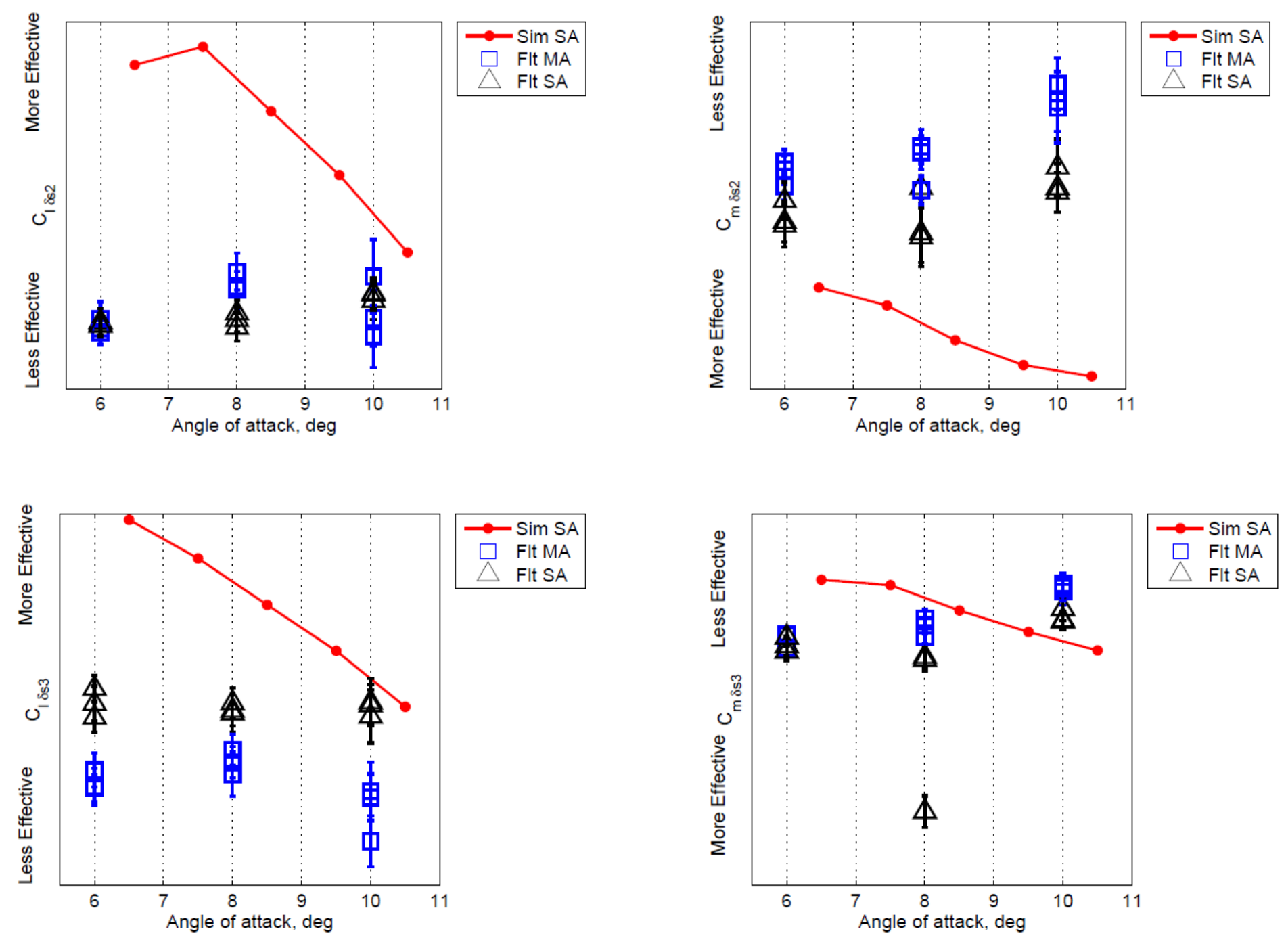


\section{Roll/pitch derivs. (Sfcs 4,5)}
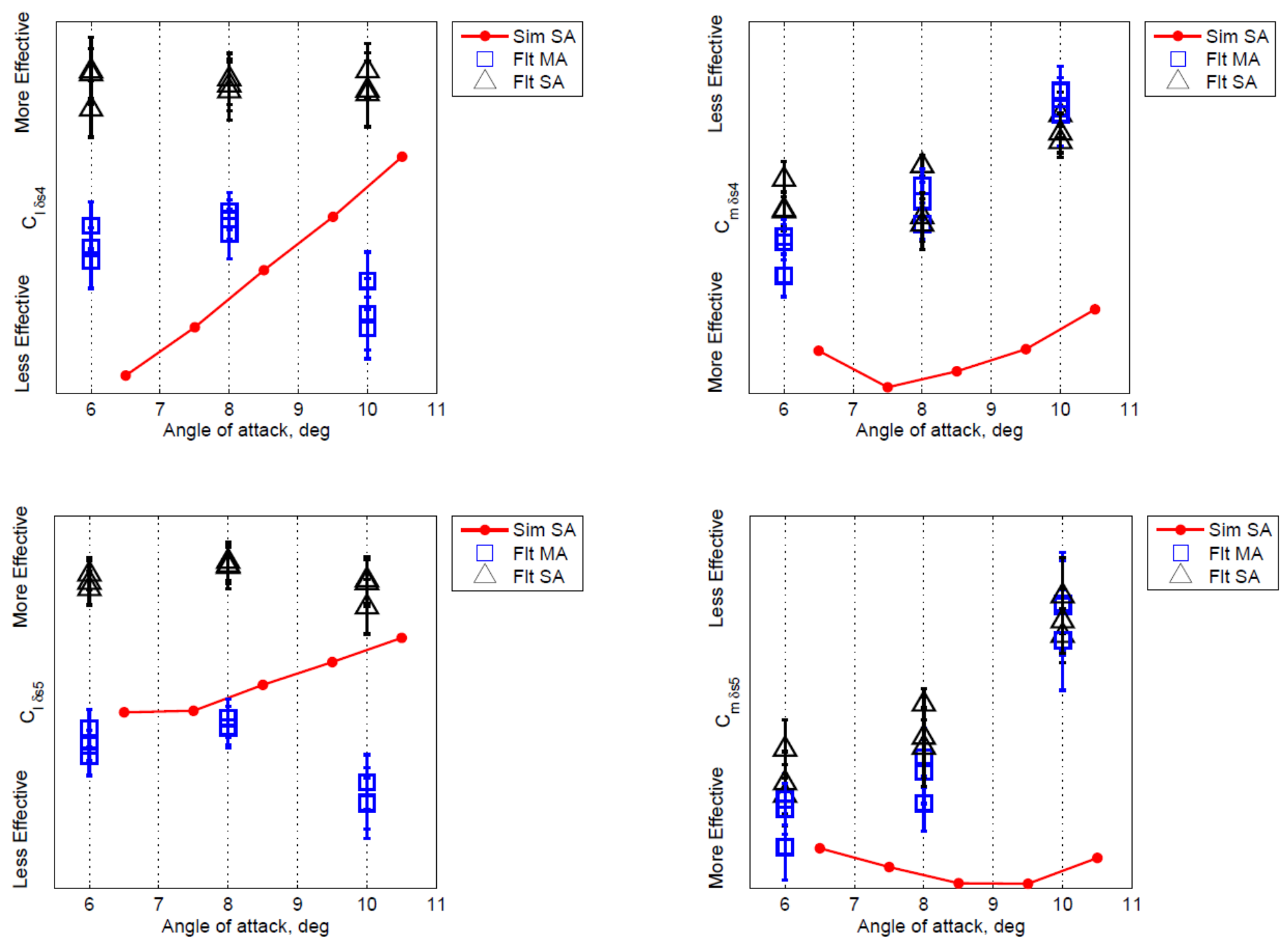


\section{Time History Reconstructions}
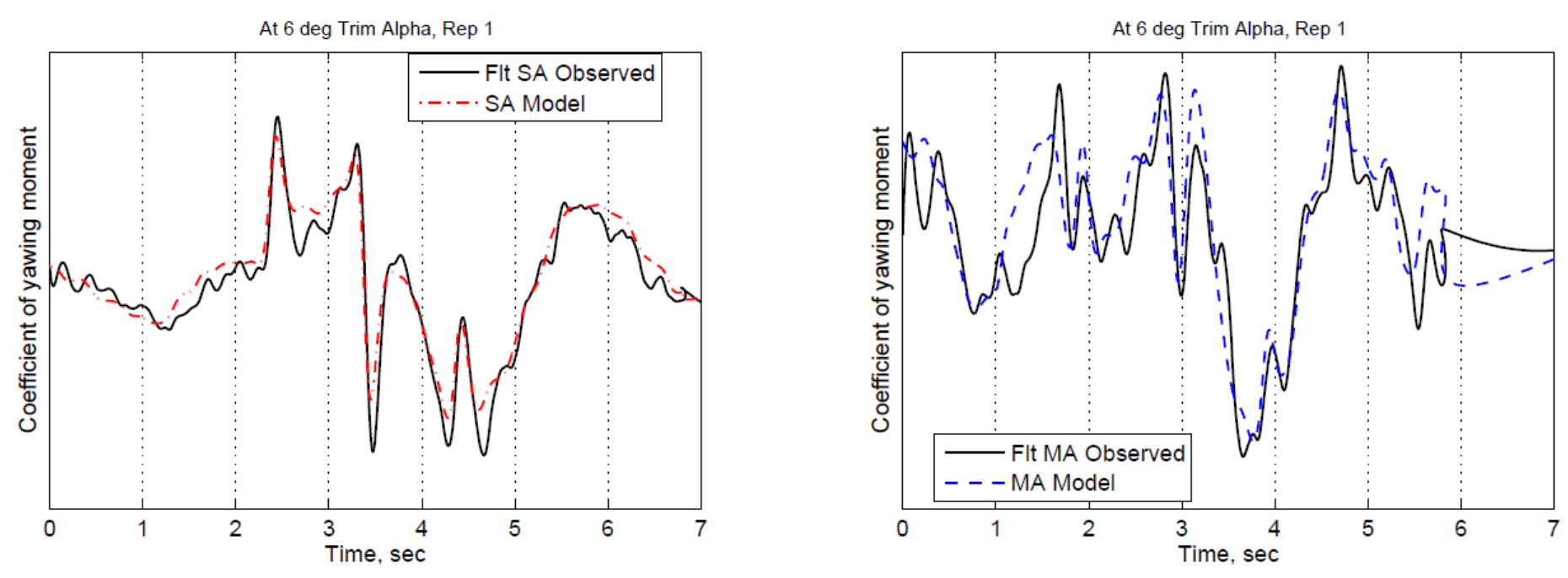

$C_{N}$
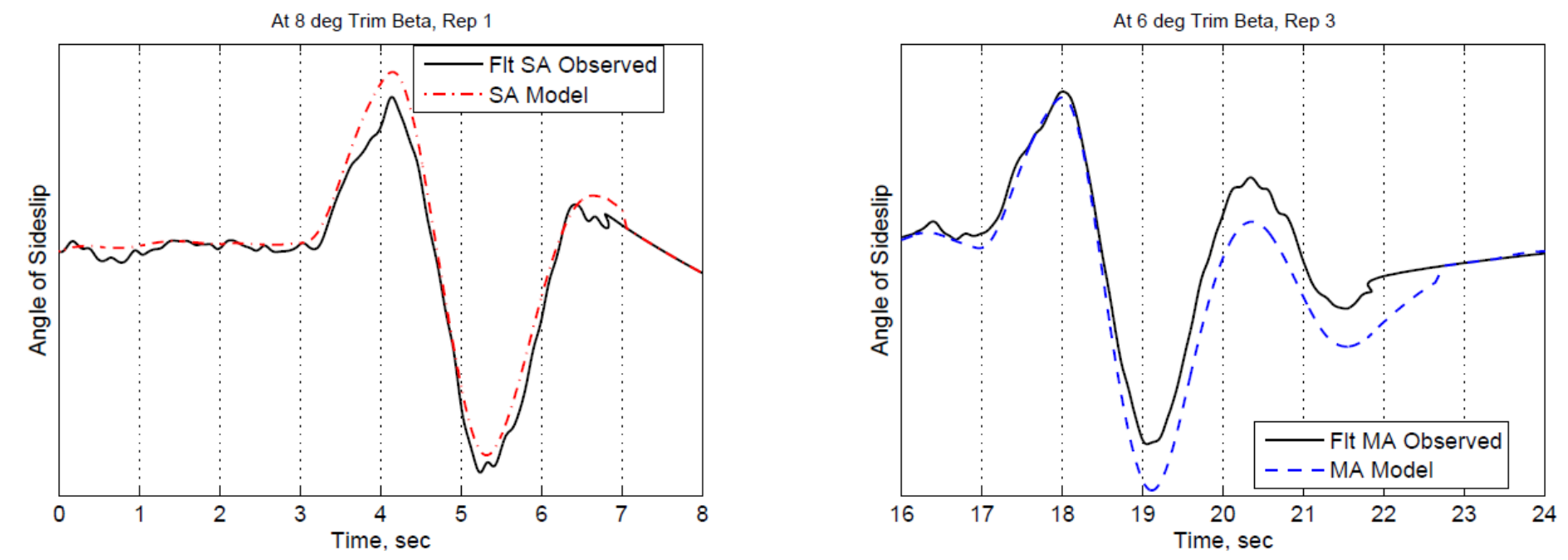

$\beta$ 


\section{Conclusions}

- Trends in multi-axis parameter estimates were quite similar to single-axis

- Typical absolute average difference in individual parameters: $15-20 \%$

- Typical multi-axis method increase in model fit error: $+1-5 \%$

- Multi-axis method exhibited greater CRB/ scatter at higher angles of attack

- Multi-axis method (as studied here) could have reduced flight test points by $50 \%$ 


\section{Questions?}
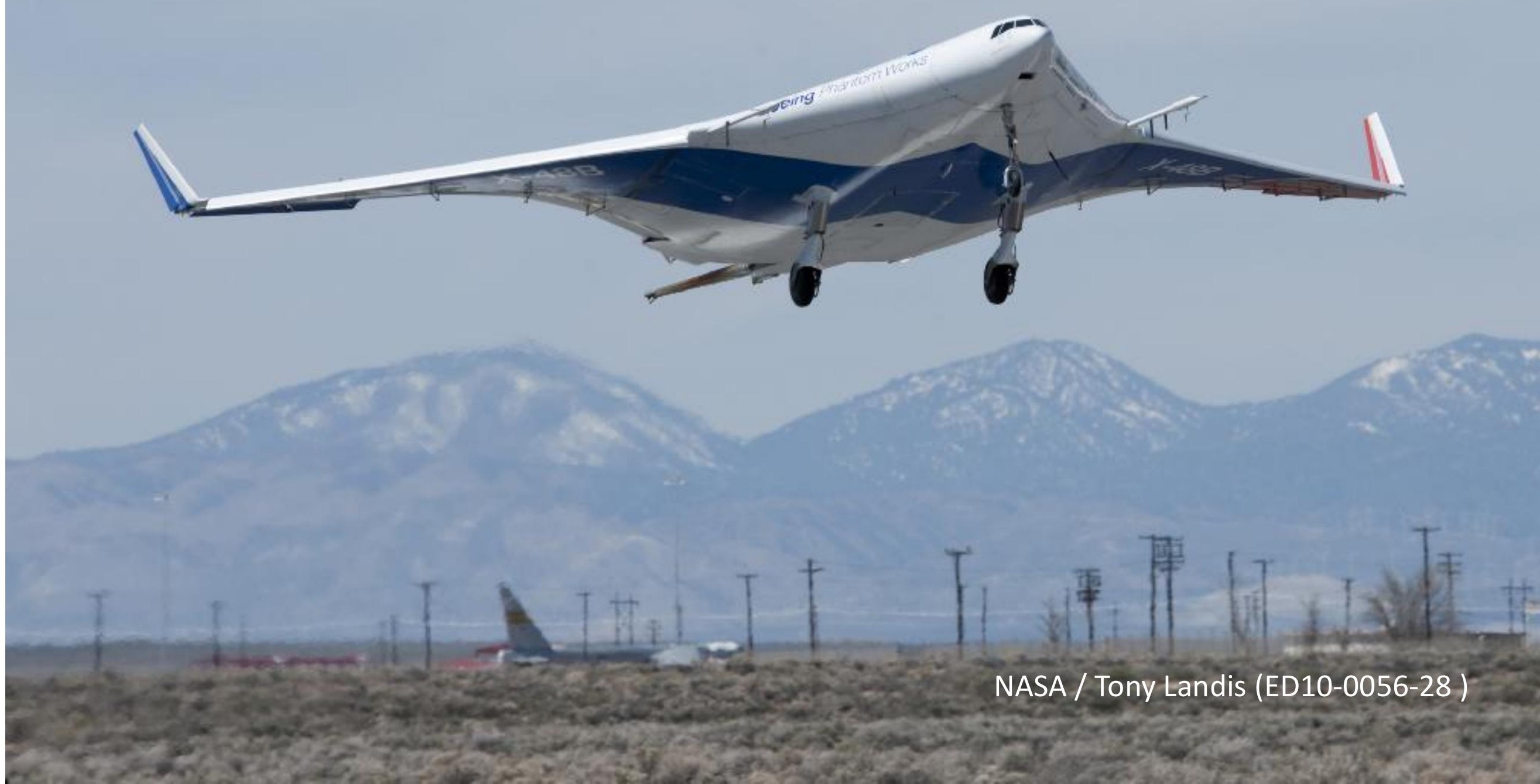